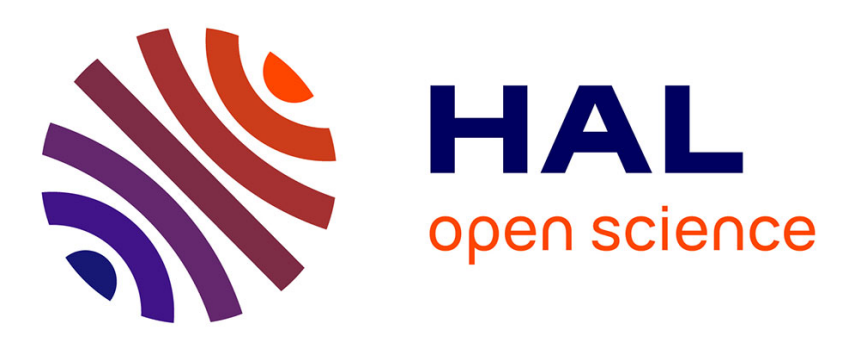

\title{
Out-of-plane elastic constants of curved cell walls honeycombs
}

Amine Harkati, El-Hadi Harkati, Abderrezak Bezazi, Fabrizio Scarpa, Morvan Ouisse

\section{- To cite this version:}

Amine Harkati, El-Hadi Harkati, Abderrezak Bezazi, Fabrizio Scarpa, Morvan Ouisse. Out-of-plane elastic constants of curved cell walls honeycombs. Composite Structures, 2021, 268, pp.113959 (13). hal-03359924

\section{HAL Id: hal-03359924 \\ https://hal.science/hal-03359924}

Submitted on 30 Sep 2021

HAL is a multi-disciplinary open access archive for the deposit and dissemination of scientific research documents, whether they are published or not. The documents may come from teaching and research institutions in France or abroad, or from public or private research centers.
L'archive ouverte pluridisciplinaire HAL, est destinée au dépôt et à la diffusion de documents scientifiques de niveau recherche, publiés ou non, émanant des établissements d'enseignement et de recherche français ou étrangers, des laboratoires publics ou privés. 


\title{
Out-of-plane elastic constants of curved cell walls honeycombs
}

\author{
Amine Harkati ${ }^{1}$, El Heddi Harkati ${ }^{1}$, Abderrezak Bezazi $^{2}$, Fabrizio Scarpa ${ }^{3}$, Morvan Ouisse ${ }^{4}$ \\ ${ }^{1}$ Université de Larbi Tébessi, Laboratoire des mines, route de Constantine, Tébessa 12002, Algérie. \\ ${ }^{2}$ Laboratoire Mécanique appliquée des nouveaux matériaux (LMANM), B.P 401 Université 8 Mai 1945 Guelma, Algérie. \\ ${ }^{3}$ Bristol Composites Institute (ACCIS) and Dynamics and Control Research Group (DCRG), University of Bristol, BS8 \\ 1TR, Bristol, UK. \\ ${ }^{4}$ Université de Bourgogne Franche Comté, FEMTO-ST Institute, CNRS/UFC/ENSMM/UTBM, Department of Applied \\ Mechanics, 24 Rue de L'épitaphe, 25000, Besançon, France.
}

\begin{abstract}
The work describes the out-of-plane properties of a curved wall honeycomb structure evaluated using analytical models and finite elements techniques. Out-of-plane properties are calculated using a theoretical approach based on energy theorems and validated using a fullscale finite element technique to simulate transverse shear tests. The effects of the curvature of the walls and the depth of the honeycomb cells on the out-of-plane elastic constants are evaluated and excellent agreement is observed between theoretical and numerical models. These curved cell wall honeycombs feature specific (i.e., relative density weighted) highly tailorable upper shear bounds that shift their maximum values with the radiuses of the curved cell walls at different internal cell angles. Finally, it is also shown that these honeycombs exhibit a particular topology with a specific upper boundary independent of the non-zero curvature cell wall adopted and only dependent upon the internal cell angle.
\end{abstract}

Keyword: honeycomb, homogenization, curvature walls, elasticity moduli, auxetic, refined model

NOMENCLATURE

$V \quad:$ Cell walls volumes.

$r \quad$ : radius of the curved cell wall.

$E_{3}$ : Young's modulus along direction 3

$t \quad$ : Cell wall thickness.

$G_{13} \& G_{23}$ : Out-of-plane shear moduli

$c:$ Length of the curved part

$G_{s}:$ Shear modulus of the core material

$b \quad:$ Depth of the cell

$v_{s} \quad$ : Poisson's ratio of the core material.

$E_{\mathrm{s}} \quad$ : Young's modulus of the core material.

$\alpha$ : curvature ratio, $(\alpha=r / l)$.

$\gamma_{13} \& \gamma_{23}:$ Shear deformations

$\tau_{13} \& \tau_{23}:$ Shear stress

$v_{23}, v_{32}$ : Out-of plane Poisson's ratios.

$\beta$ : Cell wall aspect ratio, $(\beta=a / l)$.

$\gamma:$ Cell wall thickness ratio, $(\gamma=t / l)$

$l \quad: \quad$ Cell wall length

$\theta \quad$ : Cell internal angle. 


\section{Introduction}

Honeycombs are discrete cellular materials that can be placed as a core between two thin composite skins and form sandwich structures. The modelling of their effective properties is of paramount importance in predicting their overall mechanical response and designing efficient sandwich structural components. The modelling of the engineering constants of honeycomb cellular solids has been pioneered by El-Sayed et al [1], followed by Gibson and Ashby [2]. Kolpakov in 1985 developed an analytical homogenization approach to describe elastic frameworks, and was one of the first to introduce the concept of auxetic (negative Poisson's ratio) honeycomb with the butterfly shape unit cell [3]. In their seminal paper Masters and Evans in 1996 developed models based on different deformation mechanisms acting on the cell ribs (bending, stretching and hinging/shear deformation) [4].

Out-of-plane mechanical properties are especially critical to define the mechanical performance and structural integrity of sandwich panels [5]. Florence et al. [6] have demonstrated that calculating the modal density of sandwich honeycomb cylinders without taking into account the transverse shear deformation can lead to significant errors at high frequency. Open literature publications related to the transverse shear of honeycomb configurations have been available since the 1950's. Other contributions to the analysis of the transverse shear in honeycomb structures has been given by Penzien and Didriksson [7], and also later by Ueng et al, in the case of superplastically formed cores [8]. The upper and lower bounds (limits) of the transverse shear modulus are calculated by energy methods and used by Gibson and Ashby [9]. Another method for obtaining two bounds is proposed by Kelsey et al. [10]. The authors used simple expressions by the application of the displacement and unit load method together with simplifying hypotheses relating to the strain and stress systems respectively in the core, the results found are validated by bending and transverse shear tests. Grediac [11] has investigated using finite element method three cell geometries in order to study the influence of the thickness on the shear modulus and on the homogeneity of the shear stress field. A relationship providing shear modulus $G_{13}$ for thin honeycombs highlighting the influence of the core thickness is given. The transverse shear modulus of honeycombs with negative Poisson's ratio in the plane has been also evaluated by Scarpa and Tomlin [12] using numerical simulations following Grediac's steps. Those authors have shown that the real transverse shear modulus is an intermediate value, which depends on the relative density and the thickness ratio were the difference between the upper and lower limits is particularly significant for the negative values. The collapse behavior under shear and simple compression out of plane has been analyzed by Zhang and Ashby [13] who concluded that there is a good agreement between the numerical 
model and the experimental results. The influence of the core geometry on the equivalent transverse shear stiffness in a honeycomb sandwich plate has been investigated by Shi and Tong [14]. Those authors concluded that the influence of the ratio between face panel and hexagon wall thicknesses was negligible when the depth of the core was large compared to the size of the hexagonal cell.

A representative three-dimensional model of volume elements with appropriate periodic boundary conditions has been proposed by Lin et al. [15] to calculate numerically the elastic properties out-of plane of the hexagonal honeycombs with edges of cells having variable and curved thicknesses. The authors have shown that the FEA numerical results indicate that the out-of-plane shear modulus, the compressive and shear buckling resistance of regular hexagonal honeycombs are significantly affected by the distribution of solids in the edges of the cells. Malek and Gibson [16] studied the elastic behavior of periodic hexagonal honeycombs over a wide range of relative densities and cell geometries, using both analytical and numerical approaches. Stefan et al. [17] presented some general models of two- and three-dimensional finite elements allowing to study the equivalent orthotropic mechanical properties of honeycombs.

Some of the above-mentioned analytical and numerical homogenization techniques have been used to model and design various classes of centre-symmetric honeycombs. For example, square stainless steel honeycombs were fabricated and analytically modelled by Cote et al. [18] based on a uniform deformation of the cell walls were they concluded that the shear strength of square honeycombs compares favorably with that of other sandwich core topologies such as nuclei pyramidal and wavy. The properties of a new honeycomb configuration proposed by Bezazi el al. [19,20] have been evaluated using an analytical model and finite elements are described by Lira et al. [21]. The analytical models are validated using a full-scale finite element technique to simulate transverse shear tests, and the different results highlight the specific deformation mechanism of the multi-reentrant honeycomb cell. Properties of transverse shear resistance of a new honeycomb with zero Poisson's ratio (ZPR) are proposed by Lira et al. [22] where the cellular configuration is simulated by a series of finite element models which were compared to the experimental results of 3-point bending shear tests to perform a parametric study on the shear moduli in both directions. The shear stiffness of a honeycomb structure with ZPR capable of obtaining deformations in two orthogonal directions are analyzed and validated using finite element techniques by Gong et al. [23]. 
Among the models of homogenization found in the literature and which consider cells with curved walls, the model developed by Balawi and Abot [24], Meraghni et al. [25], Hohe and Becker [26,27]. The in-plane elasticity modulus of a curved-walled cell was studied by Balawi and Abot [24] using analytical and numerical means and correlated with the experimental results for aluminum honeycomb cells. Analytical and numerical models describing the uniaxial elastic properties in the plane (Young's moduli and Poisson's ratio) of a honeycomb structure with curved walls have been studied by Harkati et al. [28].

A numerical model able to predict the impact behaviour of natural fibres honeycomb cores has been proposed by [33]. The numerical results in that paper have been validated by comparisons with experimental data in terms of impact force as a function of the impactor displacement. The results have demonstrated the validity and the robustness of the proposed numerical model. The impact behavior of several sandwich composite panels configurations with polypropylene core has been described by Riccio et Al [34]. Delamination and indentation caused by low velocity impact has been evaluated in that paper in the case of several sandwich panels with varying polypropylene core materials. The impact resistance has also been associated to the fire compliance of the material cores, therefore providing a comprehensive insight on the multifunctional behavior of those sandwich composite panels. Petrone et al [35] have investigated the behaviour of two different types of polymeric cores (short flax fibre reinforced and continuous flax fibre reinforced) under low velocity out-of-plane impact loading. The Authors found a better energy absorption when honeycombs with cell walls reinforced with continuous fibres are present, while face skins play an important role during energy absorption only at low core heights. The effect of the cell shape and the foil thickness on the crushing behavior of honeycombs has also been investigated by Yamashita and Gotoh [36] by using an explicit FEM code (DYNA3D). The results show that a cyclic buckling mode takes place and that the crushing strength is larger for smaller branch angles. When the crushing strength is evaluated with respect to the net cross-section of the material part only, it attains the maximum value when the cell shape is a regular hexagon. In order to increase the impact resistance of sandwich panels, a continuum damage model has been proposed by Horrigan et al [37]. The model developed in that paper describes the compressive behaviour of honeycombs made from materials that are prone to elastic buckling. The same model has been embedded within a commercial finite element package to simulate soft body impacts onto a minimum gauge honeycomb core sandwich. 
The aim of this work is to evaluate the out-of-plane shear properties (Voight and Reuss bounds) of curved cell walls honeycombs designs. The main novelties if this investigation is the evaluation of the five out-of-plane homogenized engineering constants of curved cell wall honeycombs versus the internal geometric parameters (internal angle $\theta$, depth and curvature of the cell walls). The comparison between the analytical and numerical models shows good agreement and makes it possible to highlight the specific out-of-plane deformation mechanisms of the honeycomb cell with curved walls.

\section{Theoretical analysis - Refined analytical model}

\subsection{Honeycomb cell geometry}

The geometry of the cell shown in figure 1 can be expressed by the three walls of lengths $a, l$, and $r$, their thickness $t$ and internal cell angles $\theta$. Like other notations used in the theory of cellular materials $[28,29]$, the geometry of the unit cell is also defined by the non-dimensional parameters $\alpha=r / l, \beta=a / l$ and $\gamma=t / l$. The present cell can represent a convex honeycomb for $r=0$ with a positive angle $\theta>0$, or an auxetic cell for a negative angle $(\theta<0)$. As it will be clearer later, auxeticity is maintained for some specific values of the cell wall radius.

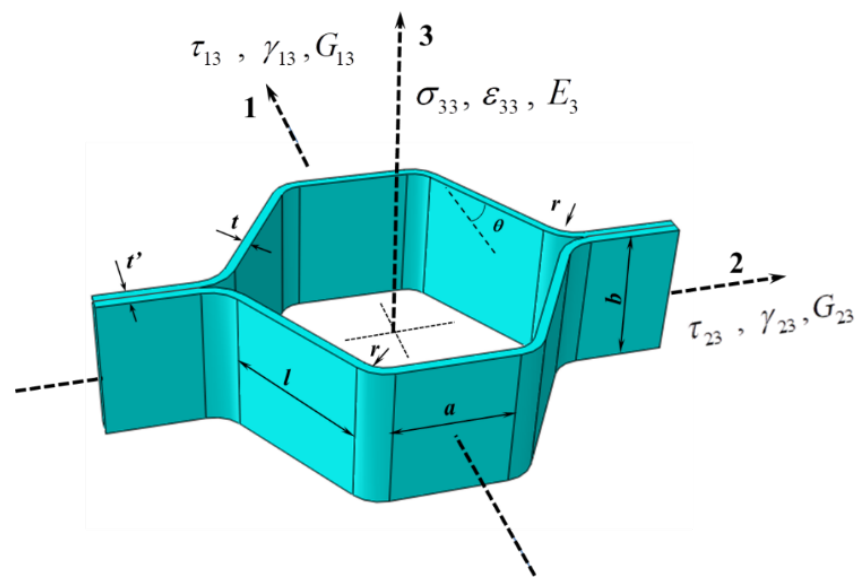

Figure 1. Geometric parameters of the honeycomb cell. 


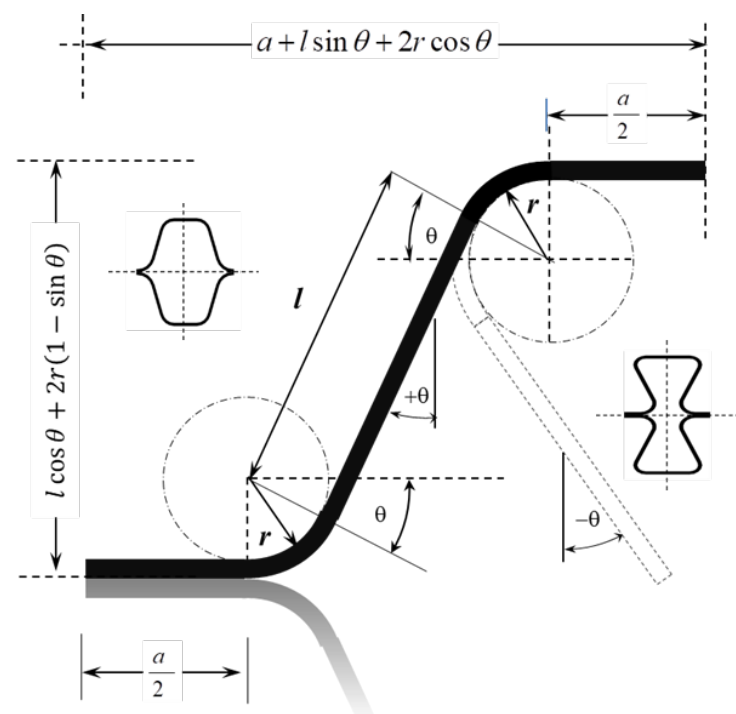

Figure 2. Geometry of a quarter honeycomb cell.

\subsection{Conditions on the geometric parameters}

The geometric limits for the cell wall to avoid touching opposite sides are of the following:

$a>2(l \sin \theta+r(1-2 \cos \theta))$

$\alpha=\frac{r}{l}, \beta=\frac{a}{l}, \gamma=\frac{t}{l}$

$\beta=\frac{a}{l}>|2(\sin \theta+\alpha(1-2 \cos \theta))|$

Those relations lead to the following sets of non-dimensional parameters:

with $-70^{\circ} \leq \theta \leq+30^{\circ}$

$\alpha=\{0,0.1, \ldots, 0.6\}$

for $\theta=-60^{\circ} \mapsto \beta=\sqrt{3} \quad \forall \frac{r}{l} \in[0,0.6] \therefore a=\sqrt{3} l$

The conditions and limits on the geometrical parameters appearing in the theoretical equations developed are represented in figure 3. The most admissible geometric parameters range occurs when inequality (1) is satisfied. The larger the $r / l(\mathrm{r} / l>0)$ ratio, the wider is the range of permissible negative internal cell angles. Note that for a zero relative curvature $(r / l=0)$ and an internal cell angle of $-30^{\circ}$ the value of $a$ must be strictly greater than $l$. For $r / l=0.6$ however, the value of $a$ is reduced to $0.12 \times l$. This value becomes greater than $1.75 l$ for an angle of -60 ${ }^{\circ}$, no matter the value of $r / l$ is. 


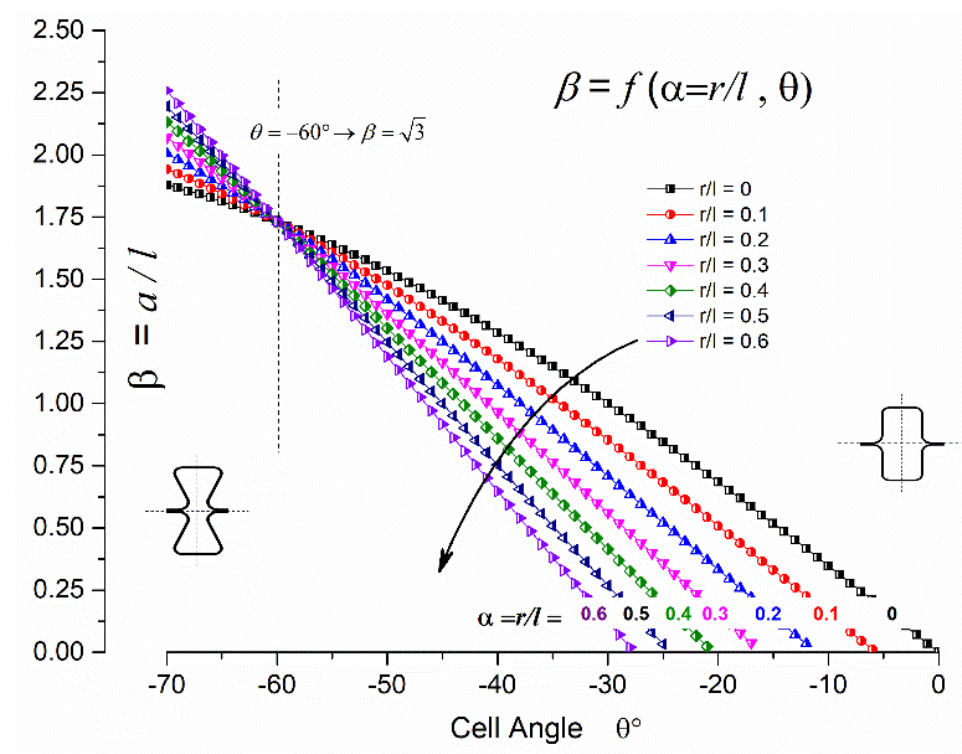

Figure 3. Possible ranges of the angles $\theta$ and $\beta$ with different aspect $r / l$ ratio.

Five engineering constants are needed to describe the out-of-plane behaviour and a total of nine for a complete description of the honeycomb. The in-plane properties of this honeycomb have been already described elsewhere [28,29].

\subsection{Determination of the transverse shear moduli}

An energy approach is used to determine the effective shear moduli of the honeycom $[9,10]$. To do this, a unit shear stress is imposed on the cell, the resulting shear flux is calculated and the corresponding shear strains are determined. By applying the unit load method and the minimum complementary energy theorem, the lower limit of the shear modulus can be found. Likewise, when a unitary shear stress is imposed on the representative element, the kinematically equivalent forces in the elements of the plate are calculated and the resulting stresses can be therefore determined. The application of the unit displacement method and the minimum potential energy theorem provides the upper limit of the shear modulus.

To apply the theoretical approach, the following assumptions are made:

- The material of the cell wall is isotropic and linear elastic;

- The thin cell walls are short, perfecty interconnected and the buckling does not occur

- The local interaction between the core and the skins is not considered 
- The estimation of the shear moduli is based on the core having small $(0<t / l<1 / 15)$ ratios, a $b / l(0<b / l<1 / 3)$ ratio sufficiently large to neglect the warping of the cell walls and a constant shear stress in each component of the unit cell;

- the thickness of the walls is uniform for all the elements of unit cells $\left(t_{l}=t_{a}=t\right)$.

\subsection{Effective out-of-plane shear modulus $G_{13}$}

Let us consider a uniform shear deformation $\gamma_{13}$ caused by a shear stress $\tau_{13}$ applied along direction 1 in the face perpendicular to face 3 (Figure 4). In this case, almost all of the elastic deformation energy of the honeycomb cell can be considered dissipated within the shear deformation of the cell walls, the bending stiffness and its energies associated being significantly smaller [22].

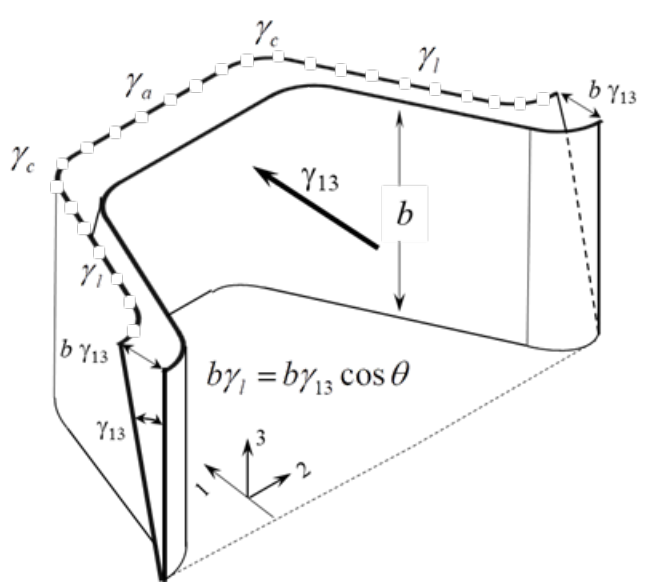

a)

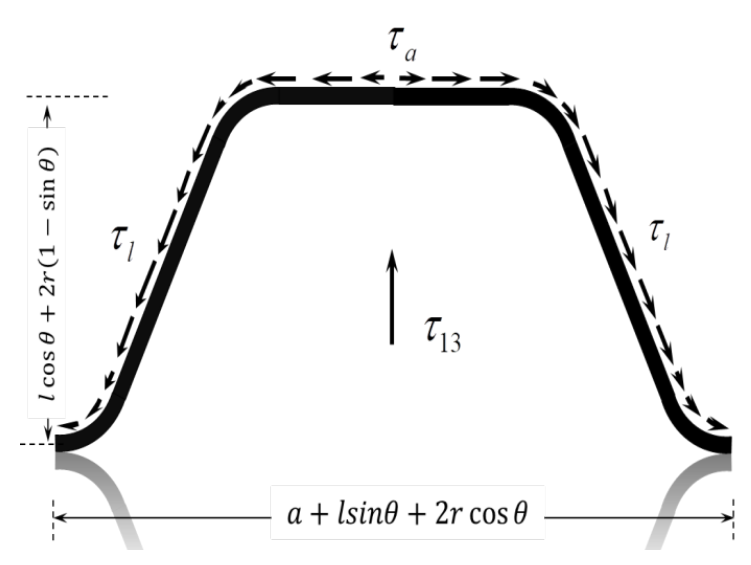

b)

Figure 4. Half honeycomb cell:

a) Shear strains due to shear deformation $\gamma_{13}$, b) Shear stress $\tau_{13}$.

The minimal potential energy theorem is therefore can be written as an inequality when shearing along the 1-direction:

$\frac{1}{2} G_{13} \gamma_{13}^{2} V \leq \frac{1}{2} \sum_{i} G_{s} \gamma_{i}^{2} V_{i} \quad$ Where $i=a, \mathrm{c}, l$

$c:$ the length of the curved part $\quad r\left(\frac{\pi}{2}-\theta\right)$

In Eq.3 the summation is carried over the cell walls with volumes $V_{a}, V_{c}$ and $V_{l}$. The induced shear strains in the cell walls (see Figure 4a) are: 
$\gamma_{l}=\gamma_{c}=\gamma_{13} \cos \theta$

$\gamma_{a}=0$

The strain energy in the curved part $c$ can be rewritten as:

$\frac{1}{2} G_{13} \gamma_{13}^{2} V=\frac{1}{2} G_{s}\left(2 \int \gamma_{l}^{2} d V_{l}+4 \int \gamma_{c}^{2} d V_{c}\right)$

With

$\int \gamma_{l}^{2} d V_{l}=b l t \gamma_{13}^{2} \cos ^{2} \theta$

To find an expression for the strain energy of the $c$ segment we consider here infinitesimal segments of length $d s=r d \varphi$ (Figure 5).

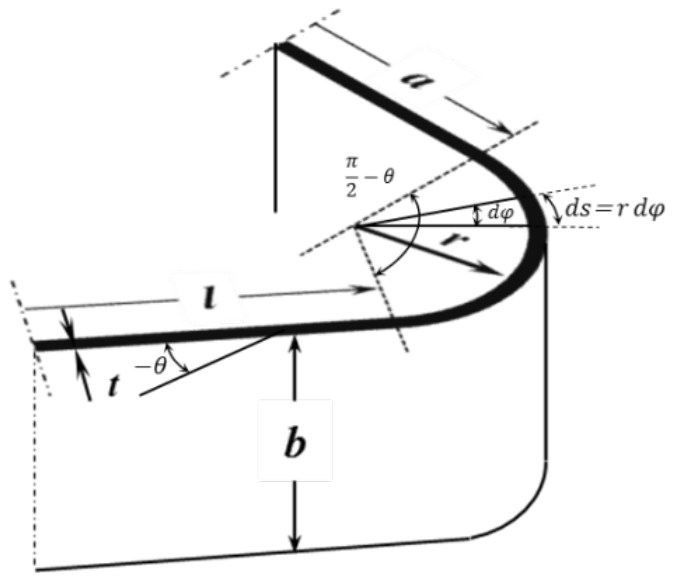

Figure 5: Geometric parameters of the curved part.

The strain energy in curved walls in part $c$ can be rewritten as:

$d V_{c}=b t d s=b t r d \varphi$

$\int \gamma_{c}^{2} d V_{c}=\int_{0}^{\frac{\pi}{2}-\theta} \gamma_{13}^{2} b t r \cos ^{2} \varphi d \varphi=b \frac{r t}{4} \gamma_{13}^{2}(\pi-2 \theta+\sin 2 \theta)$

The shear strain energy inequality can be therefore written as:

$G_{13} \gamma_{13}^{2} V \leq 2 b t G_{s} \gamma_{13}^{2}\left(l \cos ^{2} \theta+\frac{r}{2}(\pi-2 \theta+\sin 2 \theta)\right)$

with the cell volume eaqual to:

$V=S b=2 b(a+2 r \cos \theta+l \sin \theta)(2 r-2 r \sin \theta+l \cos \theta)$

Finally, the expression of the upper shear bound for the modulus $G_{13}$ becomes: 
$\frac{G_{13}}{G_{s}} \leq \gamma \frac{\cos ^{2} \theta+\frac{1}{2} \alpha(\pi-2 \theta+\sin 2 \theta)}{(\beta+\sin \theta+2 \alpha \cos \theta)(\cos \theta+2 \alpha(1-\sin \theta))}$

If we set $r=0$, we obtain the expression of the shear modulus of a conventional cell (see Gibson and Ashby [9]):

$$
\frac{G_{13}}{G_{s}} \leq \gamma \frac{\cos \theta}{\beta+\sin \theta}
$$

To find the lower bound of the effective transverse shear modulus $G_{13}$, an external stress $\tau_{13}$ is applied to the faces of the cell. The stress induces a shear flow in the honeycomb cell walls (Figure 4.b). The straight wall $a$ is under bending deformation only and therefore $\tau_{a}=0$.

$$
\frac{1}{2} \frac{\tau_{13}^{2}}{G_{13}} V \leq \frac{1}{2} \sum_{i} \frac{\tau_{i}^{2}}{G_{s}} V_{i} \quad i=a, \mathrm{c}, l
$$

The equilibrium forces along the 1 direction require that the applied shear force on the unit cell must be equal to the sum of the shear forces.

$$
\tau_{13} V=2 \tau_{l} t(l \cos \theta+2 r(1-\sin \theta)) b
$$

From where

$$
\tau_{l}=\frac{\tau_{13} V}{2 b t(l \cos \theta+2 r(1-\sin \theta))}
$$

The complementary energy inequality becomes:

$$
\frac{\tau_{13}^{2}}{G_{13}} V \leq 2 \frac{\tau_{l}^{2}}{G_{s}}\left(l+2 r\left(\frac{\pi}{2}-\theta\right)\right) b t
$$

With the expression of $V$ given in (6), the lower bound of the effective transverse shear modulus $G_{13}$ of the honeycomb can be finally expressed as:

$$
\frac{G_{13}}{G_{s}} \geq \gamma \frac{\cos \theta+2 \alpha(1-\sin \theta)}{(\beta+2 \alpha \cos \theta+\sin \theta)(1+\alpha(\pi-2 \theta))}
$$


Again, if $r=0$ we obtain to the lower bound of the shear modulus $G_{13}$ of a conventional cell equation (8) (see Gibson and Ashby [9]).

\subsection{Effective out-of-plane shear modulus $G_{23}$}

We now consider a shear stress $\tau_{23}$ acting on the surface of the normal to the 3-direction and along direction 2 (Figure 6). The induced shear strains in the cell walls can be expressed as:

$$
\begin{aligned}
& \gamma_{l}=\gamma_{c}=\gamma_{23} \sin \theta \\
& \gamma_{a}=\gamma_{23} \sin \theta
\end{aligned}
$$

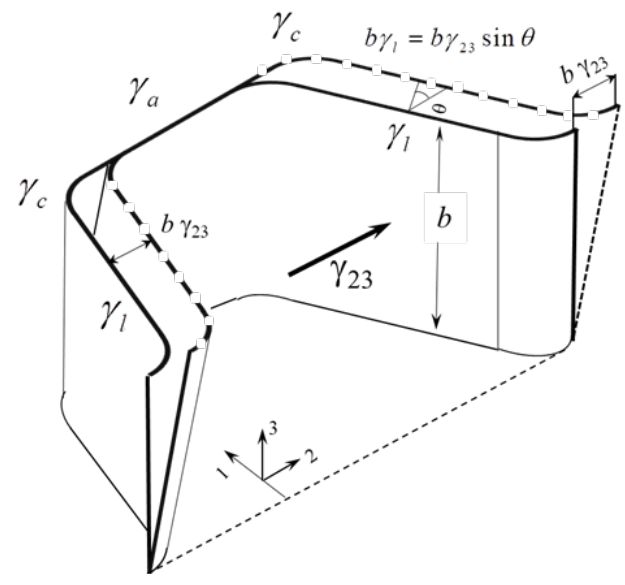

a)

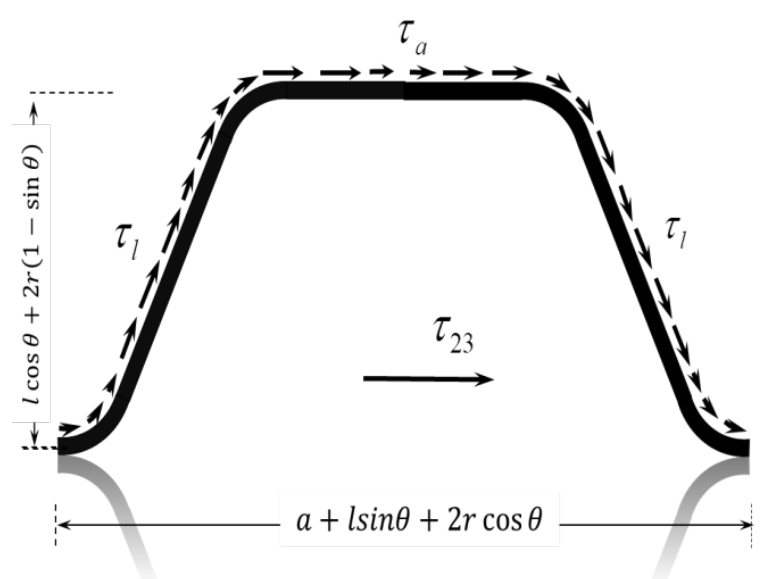

b)

Figure 6. Half honeycomb cell:

a) Shear strains due to shear deformation $\gamma_{13}$, b) Shear stress $\tau_{13}$.

The theorem of minimal potential energy is expressed as the following inequality:

$$
\begin{aligned}
& \frac{1}{2} G_{23} \gamma_{23}^{2} V \leq \frac{1}{2} \sum_{i} G_{s} \gamma_{i}^{2} V_{i} \quad i=a, \mathrm{c}, l \\
& \frac{1}{2} G_{23} \gamma_{23}^{2} V \leq \frac{1}{2} G_{s}\left(\int \gamma_{a}^{2} d V_{a}+2 \int \gamma_{l}^{2} d V_{l}+4 \int \gamma_{c}^{2} d V_{c}\right)
\end{aligned}
$$

With:

$$
\begin{aligned}
& \int \gamma_{l}^{2} d V_{l}=\gamma_{23}^{2} l t \sin ^{2} \theta \\
& d V_{c}=b t r d \varphi \\
& \int \gamma_{c}^{2} d V_{c}=\int_{0}^{\frac{\pi}{2}-\theta} \gamma_{23}^{2} b t r \sin ^{2} \varphi d \varphi=b \frac{r t}{4} \gamma_{23}^{2}(\pi-2 \theta-\sin 2 \theta)
\end{aligned}
$$

Substituent (14) in the equation (13), we can obtain: 


$$
\begin{aligned}
& G_{23} \gamma_{23}^{2} V \leq b t G_{s} \gamma_{23}^{2}\left(a+2 l \sin ^{2} \theta+r(\pi-2 \theta-\sin 2 \theta)\right) \\
& \frac{G_{23}}{G_{s}} \leq \frac{1}{2} \gamma \frac{\beta+2 \sin ^{2} \theta}{(\beta+\sin \theta) \cos \theta}
\end{aligned}
$$

For shear loading in the 2-3 plane, the applied shear stress induces shear forces in the cell walls that should be in equilibrium with the applied shear force along the 2-direction:

$$
\begin{aligned}
& \tau_{23} V=2 \tau_{l} t(a+l \sin \theta+2 r \cos \theta) b \\
& \left.\frac{1}{2} \frac{\tau_{23}^{2}}{G_{23}} V \leq \frac{1}{2} \sum_{i} \frac{\tau_{i}^{2}}{G_{s}} V_{i}\right\} i=c, l, a \\
& \tau_{l}=\frac{\tau_{13} V}{2 b t(a+l \sin \theta+2 r \cos \theta)} \\
& V=S b=2 b(a+2 r \cos \theta+l \sin \theta)(2 r-2 r \sin \theta+l \cos \theta)
\end{aligned}
$$

Finally, the effective lower shear modulus as:

$$
\frac{G_{23}}{G_{s}} \geq \gamma \frac{\beta+\sin \theta+2 \alpha \cos \theta}{\left(1+2 \beta+2 \alpha\left(\frac{\pi}{2}-\theta\right)\right)(\cos \theta+2 \alpha(1-\sin \theta))}
$$

Expression (16) and (18) do not depend on the depth $b$ of the cells. If we set $r=0$, we return to the expression of the lower bound of the shear modulus $G_{23}$ of a conventional honeycomb (Gibson and Ashby [9]).

$$
\frac{G_{23}}{G_{s}} \geq \gamma \frac{\beta+\sin \theta}{(1+2 \beta) \cos \theta}
$$

\subsection{Unit load and displacement models}

The upper and lower bounds of the honeycomb sandwich core shear modules can also be following the methodology of Kelsey et al. [10] by applying the unit displacement and load methods on a different topology of the elementary cell (Figure 7), together with simplifying assumptions about the deformations and stresses existing in the core.

Off-the-shelf manufactured honeycomb cores possess[9] double thickness horizontal walls and can be built up by repeating the unit cell shown in Figure $7[10]$. 


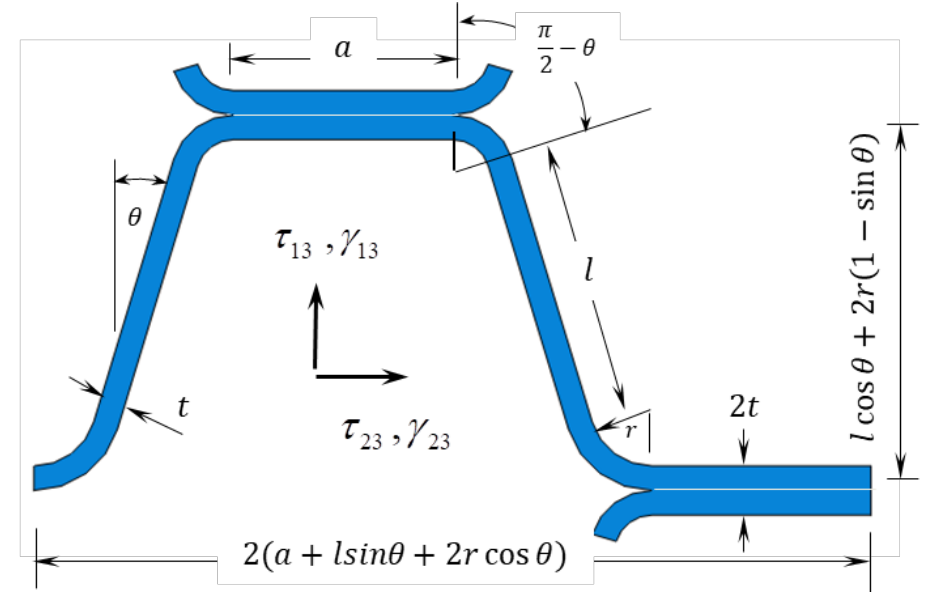

Figure 7. The unit-elements of Kelesy et al. [10] model.

In this case $\mathrm{t}^{\prime}=2 \mathrm{t}$ and the expressions of the bounds change as follows:

$$
\begin{aligned}
& \frac{G_{23}^{\text {Lower }}}{G_{s}}=2 \gamma \frac{(\beta+\sin \theta+2 \alpha \cos \theta)^{2}}{(1+\beta+\alpha(\pi-2 \theta)) S} \\
& \frac{G_{23}^{\text {Upper }}}{G_{s}}=\gamma \frac{\left(\beta+\sin ^{2} \theta\right)+\frac{1}{2} \alpha(\pi-2 \theta-\sin 2 \theta)}{S}
\end{aligned}
$$

With

$$
S=2(\beta+2 \alpha \cos \theta+\sin \theta)(2 \alpha+\cos \theta-2 \alpha \sin \theta)
$$

\subsection{Out-of-plane Poisson's ratio}

It is assumed here that $E_{3}>>E_{1}$ when a stress is applied along its depth length. The stress will result in axial deformations, while direct stresses applied in the plane will produce deformations dominated by the bending of the internal walls. The Poisson's ratios can be expressed as $[9,10]$ :

$$
\begin{aligned}
& v_{13}=v_{31} \frac{E_{1}}{E_{3}} \approx 0 \\
& v_{23}=v_{32} \frac{E_{2}}{E_{3}} \approx 0
\end{aligned}
$$

Other assumptions for the Poisson's ratios in the planes containing the 3-direction are (Gibson and Ashby [9]):

$$
v_{31}=v_{32}=v_{s}
$$




\subsection{Out-of-plane Young's Modulus $E_{3}$}

The out-of-plane Young's modulus of the honeycombs in the axial or 3-direction can be estimated by assuming that for the normal loading along the 3 direction, the deformation is axial. Therefore, the $E_{3}$ of the honeycomb can be expressed as:

$$
\sigma_{3}=\frac{v_{13}}{1-v_{12}-2 v_{13} v_{31}} E_{3}\left(\varepsilon_{1}+\varepsilon_{2}\right)+\frac{1-v_{12}}{1-v_{12}-2 v_{13} v_{31}} E_{3} \varepsilon_{3}
$$

$v_{13}=v_{23} \approx 0$

The stress acting on the area occupied by a unit cell can be simply expressed as:

$$
\sigma_{3}=E_{3} \varepsilon_{3}
$$

Therefore:

$$
\begin{aligned}
& \frac{E_{3}}{E_{s}}=\frac{\rho}{\rho_{s}}=\gamma\left\{\frac{1}{2} \frac{\beta+2+2 \alpha(\pi-2 \theta)}{(\beta+2 \alpha \cos \theta+\sin \theta)(2 \alpha+\cos \theta-2 \alpha \sin \theta)}\right\} \rightarrow t^{\prime}=\mathrm{t} \\
& \frac{E_{3}}{E_{s}}=\frac{\rho}{\rho_{s}}=\gamma \frac{(\beta+1+\alpha(\pi-2 \theta))}{(\beta+2 \alpha \cos \theta+\sin \theta)(2 \alpha+\cos \theta-2 \alpha \sin \theta)} \rightarrow t^{\prime}=2 \mathrm{t}
\end{aligned}
$$

The Young's modulus $E_{3}$ along the normal loading in the 3 direction simply reflects the solid modulus Es scaled by the area of the load-bearing section. The term in the curly brackets of equation (26) merely gives a more precise description of the projected area of the cell walls normal to the 3-direction.

\section{Finite element modelling}

A simplified finite element procedure to determine the equivalent out-of-plane engineering constants of this core and also for different parametric configurations (conventional and auxetic) is presented here. Table 1 shows the boundary conditions applied to a representative elementary volume (REV) (Figure 8) in order to reproduce the global behaviour of the honeycomb core. 
Since the deformations vary in the normal direction to the plane and the honeycomb cell is periodic only in one plane, a 3D model needs to take into account the bending of the walls to accurately assess the equivalent transverse properties of the cell structure [14].

Table 1. Boundary conditions applied to simulate the out-of-plane engineering constants

\begin{tabular}{|c|c|c|c|c|}
\cline { 3 - 5 } \multicolumn{2}{c|}{} & $\mathrm{E}_{3}, \mathrm{v}_{13}, \mathrm{v}_{23}$ & $G_{13}$ & $G_{23}$ \\
\hline \multirow{3}{*}{$\mathrm{Z}=0$} & $\mathrm{U}_{1}$ & 0 & 0 & 0 \\
\cline { 2 - 5 } & $\mathrm{U}_{2}$ & 0 & 0 & 0 \\
\cline { 2 - 5 } & $\mathrm{U}_{3}$ & 0 & 0 & 0 \\
\hline \multirow{3}{*}{$\mathrm{Z}=b$} & $\mathrm{U}_{1}$ & - & 1 & 0 \\
\cline { 2 - 5 } & $\mathrm{U}_{2}$ & - & 0 & 1 \\
\cline { 2 - 5 } & $\mathrm{U}_{3}$ & 1 & - & - \\
\hline
\end{tabular}

The effective stiffness of the core is determined by finding the force necessary to produce a unit displacement along a given direction, by determining the stress and the resulting strain and finally by calculating the corresponding engineering constants. This process can be used to find the five effective out-of-plane engineering constants namely: $E_{3}, G_{13}, G_{23}, v_{13}$ and $v_{23}$ :

$$
\left\{\begin{array}{l}
E_{3}=\frac{\sigma_{3}}{\varepsilon_{3}}=\frac{b}{S} \frac{F_{3}}{u_{3}} \\
G_{i j}=\frac{\tau_{i j}}{\gamma_{i j}}=\frac{b}{S} \frac{F_{i}}{u_{i}} \quad i=1,2 ; j=3 \\
v_{i j}=-\frac{\varepsilon_{j}}{\varepsilon_{i}}
\end{array}\right.
$$

$\sigma_{3}$ and $\varepsilon_{3}$ are the normal stress and strain in 3-direction; $\tau_{i j}, G_{i j}$ and $v_{i j}$ are shear stress, shear modulus and Poisson's ratio respectively in $i-j$ plane. The FE element model represented by REV is an assembly of 53 cells modeled with the commercial code Abaqus 6.10 [30]. The REV is composed by 63975 elastic shell elements with the reduced integration technique (S4R).

The FEA results indicate that the distribution of the elements in the curved walls plays an important role to determine the out-of-plane elastic properties of these honeycombs. 


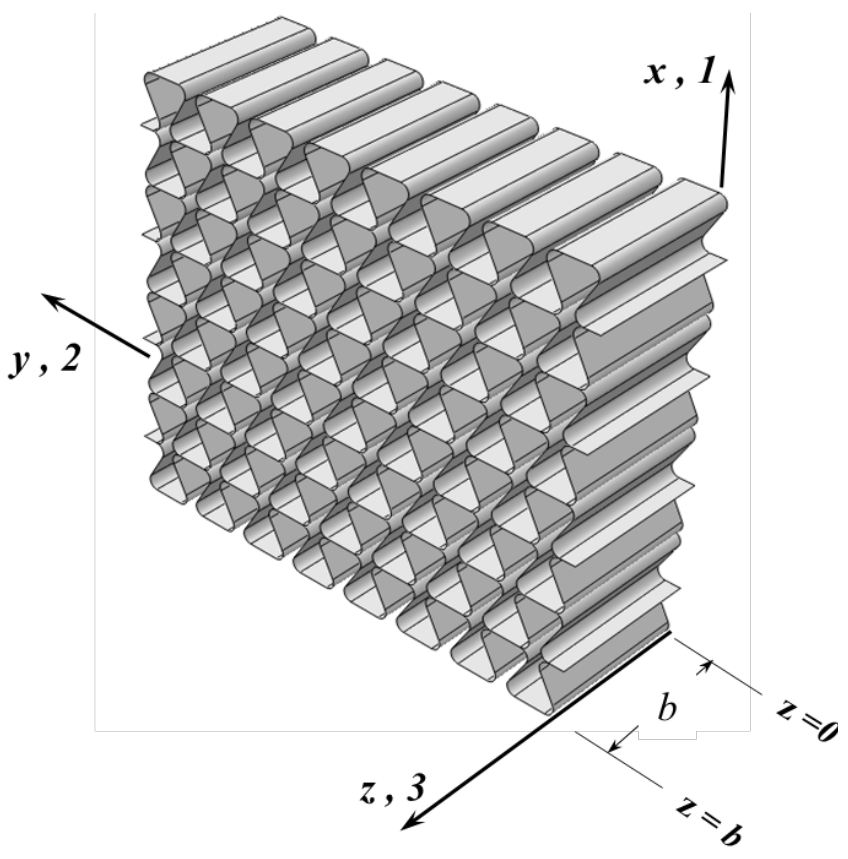

a)

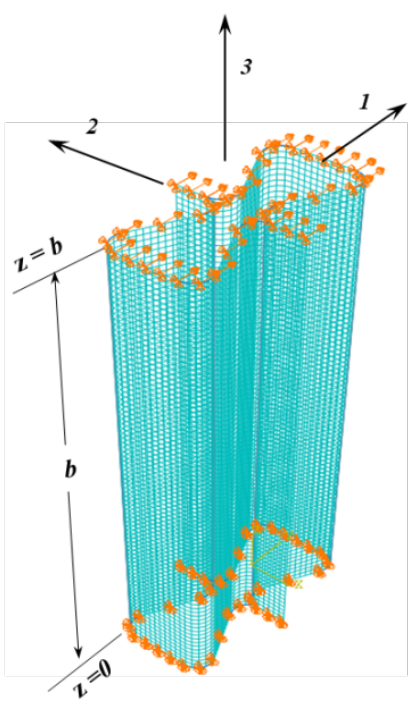

b)

Figure 8. Numerical model description: a) Representative elementary volume (REV).

b) Boundary conditions applied to simulate $G_{l 3}$.

The effect of the order of elements in the convergence of the results has been studied by considering shell elements with 4 and 8 nodes. [30]. The results were practically identical for the two elements for $E_{3}$ and a slight difference in the case of $G_{13}$ and $G_{23}$, therefore, only elements with 4 nodes are used.

The numerical models representing the shear stiffness have been refined specifically in the curved part of cell walls to represent more accurately bending effects. A comparison of the outof-plane shear stiffness with the one of classic hexagonal cells was first made to benchmark the results. An additional verification was provided by comparing directly the analytical expressions formulated.

\section{Results and discussions}

The curved cell walls are represented by arcs and the internal stress components are assumed to be constant in each member without introducing any significant error. The analytical expression of the upper bound was also obtained using the minimum total potential energy theorem, in which a stress state compatible with the boundary conditions was imposed by neglecting the stress in the honeycomb core. On the other hand, an estimate of the lower bound was obtained using the theorem of the minimum complementary energy, in which the 
equilibrium of the components of the internal stress with boundary conditions is imposed by neglecting the compatibility of the stress in the core honeycomb. It should be noted that a difference with the analytical upper and lower bounds are $10 \%$ to $35 \%$ for the modules $G_{13}$ and $G_{23}$. This is in accordance with the work of Zhang and Ashby [13] and Chamis et al [31] based on detailed three-dimensional finite element models containing several cells. These results were based on using the same boundary conditions adopted for the cells of our model. Chamis et al [31] also observed a slight difference between $G_{23}$ and $G_{32}$, which is in perfect agreement with current results as with all other types of honeycomb cells. The out-of-plane stiffness turns out to be linearly proportional to the relative density [28]. The out-of-plane Poisson's ratio is estimated at approximately zero because the out-of-plane stiffness is significantly larger than the in the plane one.

\subsection{Theoretical bounds of $G_{23} / G_{s}$}

Figure 9 shows the variation of the theoretical upper and lower limits of the non-dimensional transverse shear modulus $G_{23} / G s$ versus the internal angle of the cell $\theta$ and the relative walls curvature for some fixed parameters $(\beta=a / l=1$ and $\gamma=t / / l=1 / 30)$. The transverse shear modulus $G_{23}$ has an inverse relationship with the relative curvature $r / l$ due to the effect of the bending deformation of the walls in the plane. It is important to note that the two $G_{23}$ bounds converge for positive internal angles and diverge for negative angles (auxetic configurations) and that for the various values of $r / l$. 


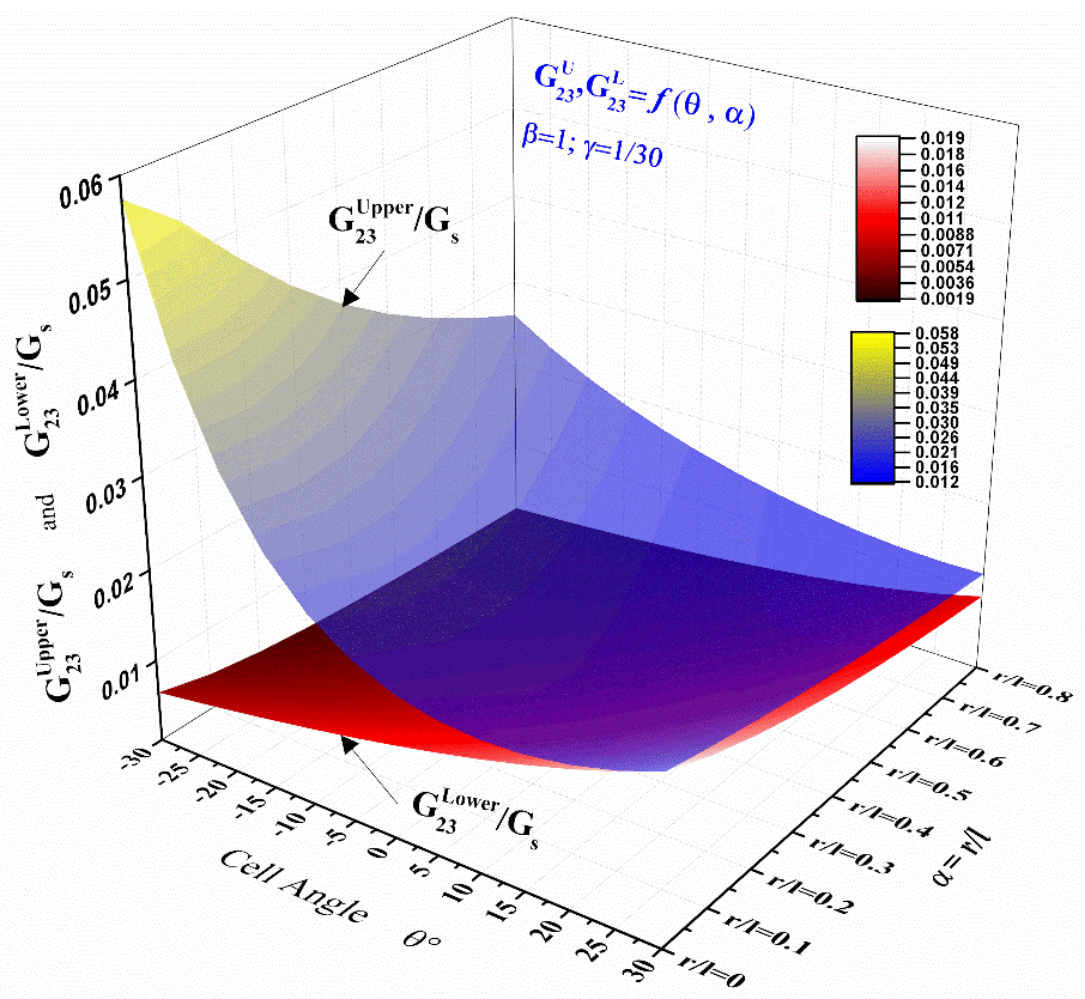

Figure 9. Upper and lower theoretical nondimensional bounds $\mathrm{G}_{23} / \mathrm{G}_{\mathrm{s}}$ versus curvature ratios $\alpha=r / l$ and internal cell angle $\theta$ (with a fixed $\beta=1, \gamma=1 / 30$ ).

\subsection{Out-of-plane shear modulus $G_{13} / G_{s}$ variation versus geometrical honeycomb parameters}

Figure 10 represents the results obtained from the variation of the out-of-plane normalized shear modulus $G_{13} / G_{s}$ versus the cell angle $\theta$. The upper and lower limits of $G_{13}$ were determined analytically and numerically by FEA for three and four values of $r / l$ curvature radius and cell depth $b / l$ respectively. The transverse shear modulus $G_{13}$ shows a strong increase in the field of negative Poisson's ratios, these variations are largely affected by the ratios $r / l$ and $b / l$. The $G_{13}$ decreases with the increase of the ratios $r / l$ and $b / l$.

The increase of $b / l$ from 1 to 10 with a fixed values of $r / l=2 / 30, a / l=1$ and $r / l=1 / 30$ leads to a $45 \%$ drop of $G_{13} / G_{s}$ for $\theta=-30^{\circ}$ and $25 \%$ for $\theta=+30^{\circ}$ (Figure $10-a$ ). For the same condition but with $r / l=10 / 30$ (Figure $10-c$ ), $G_{13} / G_{s}$ decreases instead by $31 \%$ for $\theta=-30^{\circ}$ and $23 \%$ for $\theta=+30^{\circ}$. When $b \geq 10 l$, the shear modulus $G_{13}$ is quite close to the lower limit given by equation (11), while for $b \leq 110 l$ it is near to the upper bound. The shear moduli of those two cell configurations can therefore be chosen as limits for the equivalent transverse stiffness shear of the honeycomb cell. 
The shear modulus $G_{13}$ is maximized for negative cell angles $\theta$, this may be particularly interesting for thin shell honeycomb sandwich structures mainly used in the aerospace where low weight and thickness accompanied by high rigidity are recommended.

The FEA results for the out-of-plane shear modulus $G_{13}$ show an excellent agreement with the analytical results and reflect the strong effect of the cell depth, especially for negative cell angles (Figure 10). In general, $G_{13}$ tends to decrease with increasing relative curved cell radiuses and cell depth.

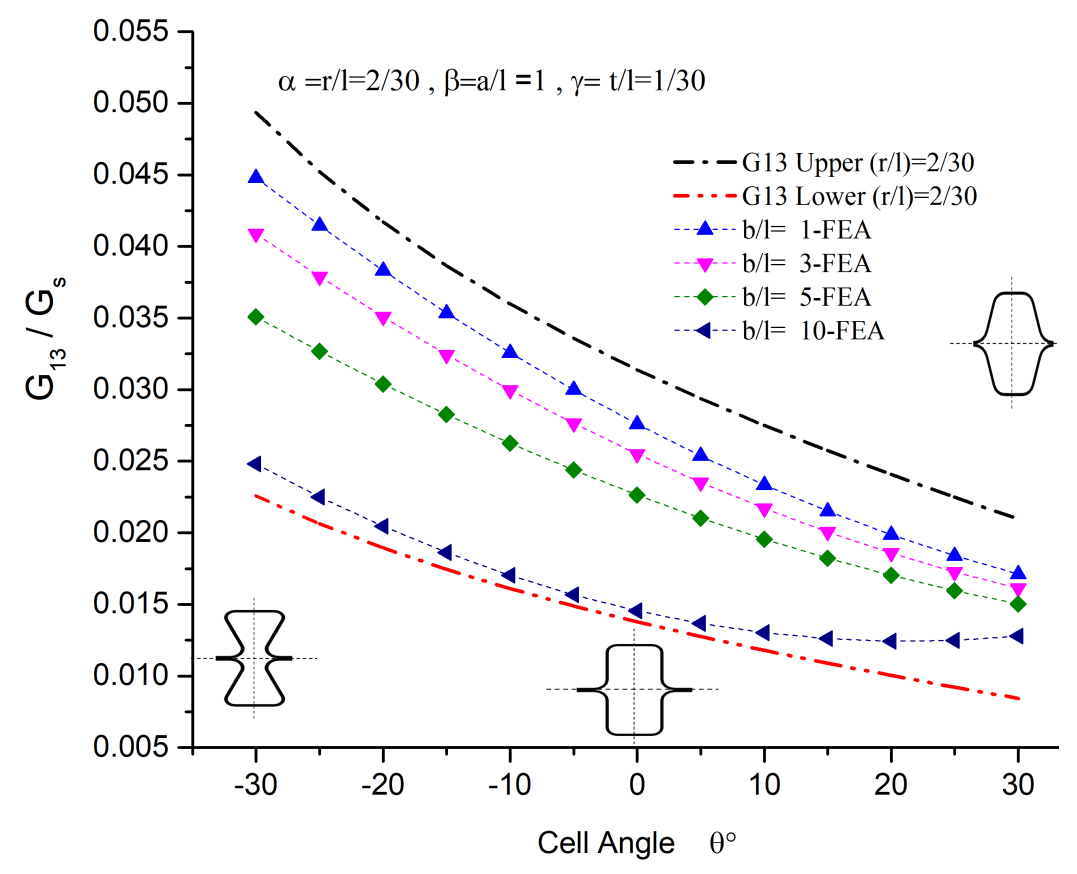

a) 


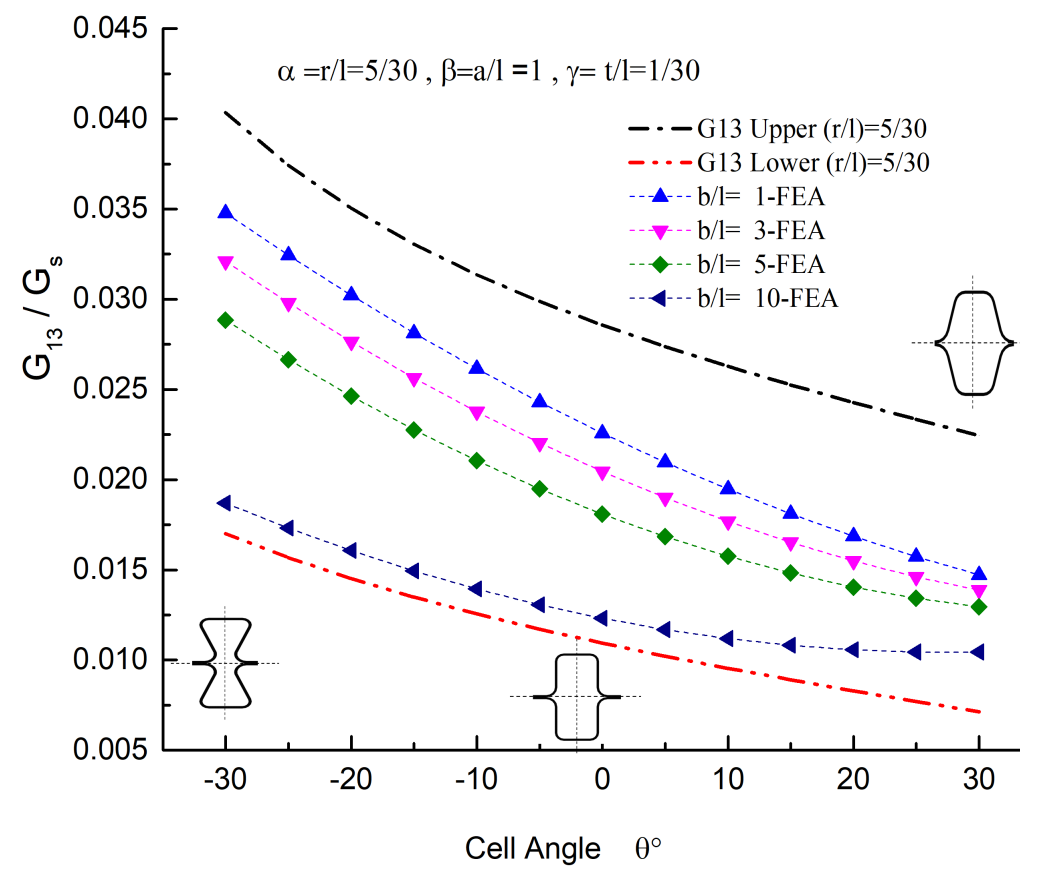

b)

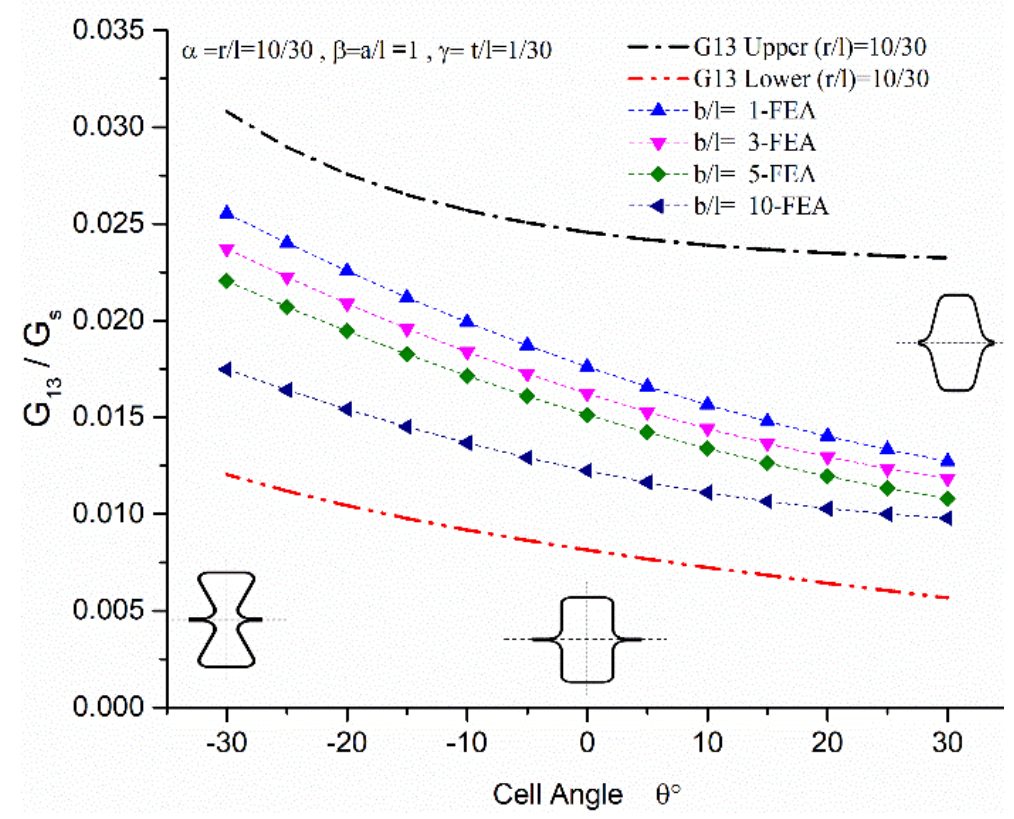

c)

Figure 10. Non-dimensional shear modulus $\left(\mathrm{G}_{13} / \mathrm{Gs}_{\mathrm{s}}\right)$ versus internal cell angle for different depths $b / l$ but with a fixed $\beta=a / l=1$ and $\gamma=t / l=1 / 30$ for: a) $\alpha=r / l=2 / 30$, b) $\alpha=r / l=5 / 30$ and c) $\alpha=r / l=10 / 30$. 
4.3 Variation of the out-of-plane shear modulus $G_{23} / G_{s}$ versus the cell effective geometric parameters

Figure 11 shows the variation of $G_{23} / G_{s}$ versus the cell angle $\theta$ for three values of curvature $(r / l=2, r / l=5$ et $r / l=10)$ and four cell depth values $b / l$. The upper and lower limits of $G_{23}$ were determined analytically. The same trend observed for $G_{13} / G_{s}$ is recorded here, nevertheless the values of $G_{23} / G_{s}$ are lower. When the radius of curvature of the wall increases, the equivalent shear modulus $G_{23}$ tends to decrease. It is clear that for a given value of $r / l$, the modulus $G_{23}$ $/ \mathrm{G}_{\mathrm{s}}$ reaches its maximum for the lowest cell depth values $(b / l<3)$ and in the case of auxetic configurations, while it decreases almost parabolically with the increase of $\theta$ (classical honeycomb).

On the other hand, the $G_{23}$ modulus of the auxetic cells is more sensitive to the $b / l$ ratio due to the effect of the bending deformation of the walls in the cell plane. An $80 \%$ drop of the transverse modulus $G_{23}$ is noticed if $b / l$ goes from 1 to 10 with $r / l=2 / 30$ and $\theta=-30^{\circ}$ (Figures 11a). The same transverse shear modulus decreases only by $20 \%$ for $\theta=+30^{\circ}$. In the case of $r / l=10$ (Figures 11c) $G_{23}$ decreases by $73 \%$ and $12 \%$ when $\theta$ is equal to -30 and +30 , respectively. The increase in $G_{23}$ is important for the small depth and cell curvature radius $(b / l$ and $r / l)$ of the auxetic configuration. The lower and upper limits of $G_{23}$ determined analytically by equations (20) and (21) are represented by the dotted lines in Figure 11 were a large difference difference between those is present for negative internal $-\theta$ cells, specially starting from $-10^{\circ}$. The FEA-determined shear modulus $G_{23}$ is relatively close to the analytical lower bound. The influence of the direction of the shear load shows that direction 1 is the best in term of shear stiffness $(>28 \%)$ for the same geometric parameters. The highest shear stiffness properties were achieved at low cell wall radiuses and for negative cell angles exceeding $-10^{\circ}$. 

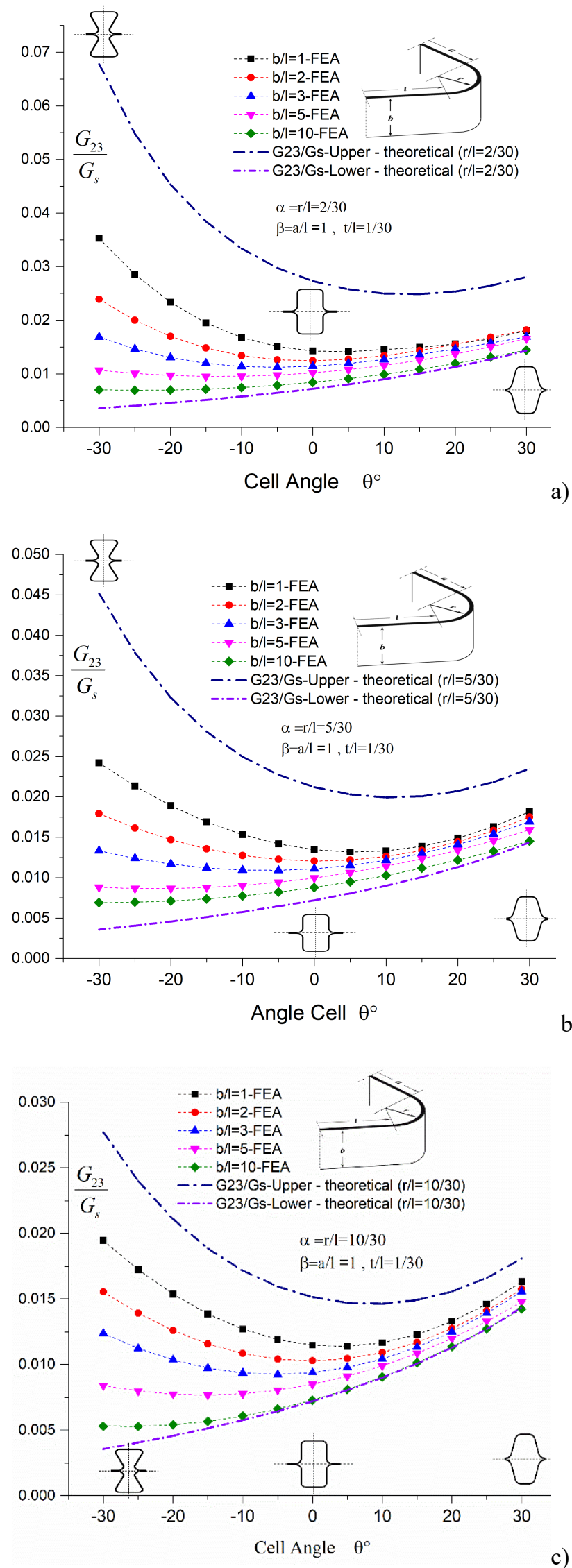

Figure 11. Non-dimensional shear modulus $\left(G_{23} / G_{s}\right)$ versus internal cell angle for different depths $b / l$ but with a fixed $\beta=a / l=1$ and $\gamma=t / l=1 / 30$ for: a) $\alpha=r / l=2 / 30$, b) $\alpha=r / l=5 / 30$ and c) $\alpha=r / l=10 / 30$. 
All the out-of-plane modules obtained using FEA for $\theta=30^{\circ}$ (Figure 11 ) tend to be $\sim 10 \%$ larger than the analytical results when $b$ tends to infinity (i.e., plane strain condition). This aspect has also been reported in [32] but only for the $\mathrm{E}_{2}$ modulus; this effect is likely due to the cell wall deformation along the 3 -direction, since $v_{\mathrm{s}}$ is not null.

\subsection{Out-of-plane shear moduli variation versus the curvature radius for different cell angles}

Figure 12 represents the evolution, in parabolic form, of the out-of-plane effective shear moduli $G_{13} / G_{s}$ and $G_{23} / G_{s}$ versus the curvature ratio $r / l$ which varies between 0 to 0.6 for different cell angles $\theta$ (from $-30^{\circ}$ to $+30^{\circ}$ at steps of $10^{\circ}$ ). The simulations are carried out by fixing the parameters $\beta=a / l=1$ and $\gamma=t / l=1 / 3$ as constant. The analysis of the results shows that the maximum values of the moduli $G_{13} / G_{s}$ and $G_{23} / G_{s}$ are obtained for $r / l$ equal to zero and the increase of this latter causes their decreases. In addition, increasing $\theta$ from -30 to +30 leads to a decrease in $G_{13} / G_{s}$ while $G_{23} / G_{s}$ increases. In other words, the maximum values of $G_{13} / G_{s}$ are obtained for conventional structures having $+\theta$, while for $G_{23} / G_{s}$ they are found for the auxetic case at $-\theta$. The increase of $r / l$ from 0 to 0.6 leads to a decrease of $G_{13} / G_{s}$ and $G_{23} / G_{s}$ by $\sim 83 \%$ and $50 \%$ respectively for an angle of $\theta=-20^{\circ}$, and by $65 \%$ and $\sim 39 \%$ for $\theta=+30^{\circ}$ (Figure 12). For a curvature ratio $r / l=0.1$, a decrease of $G_{13} / G_{s}$ and $G_{23} / G_{s}$ by $\sim 56 \%$ and $70 \%$ respectively is observed between the two extreme cell angles $\theta=-30^{\circ}$ and $\theta=+30^{\circ}$.

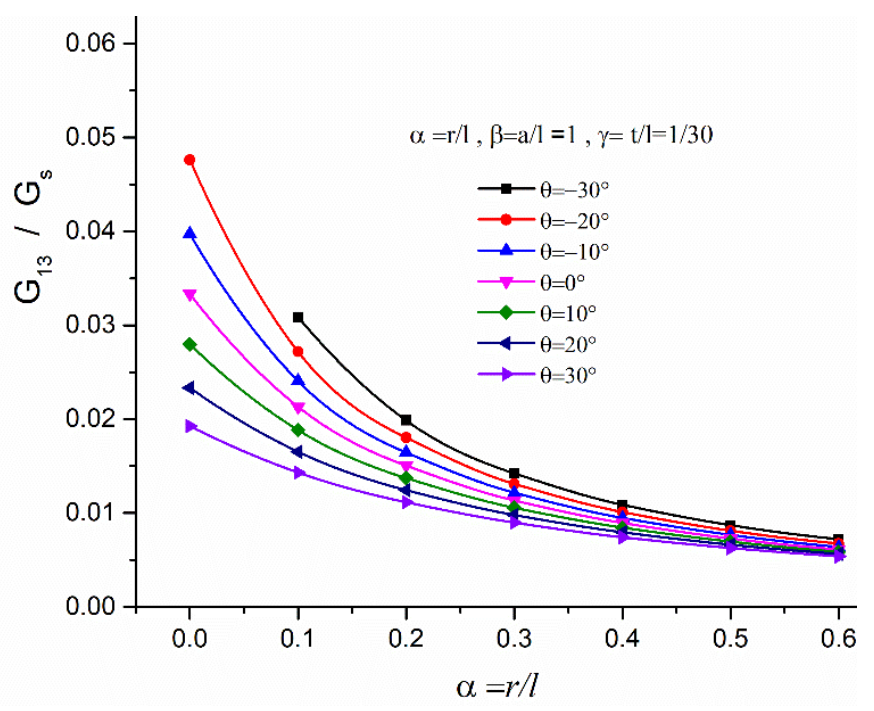

a) 


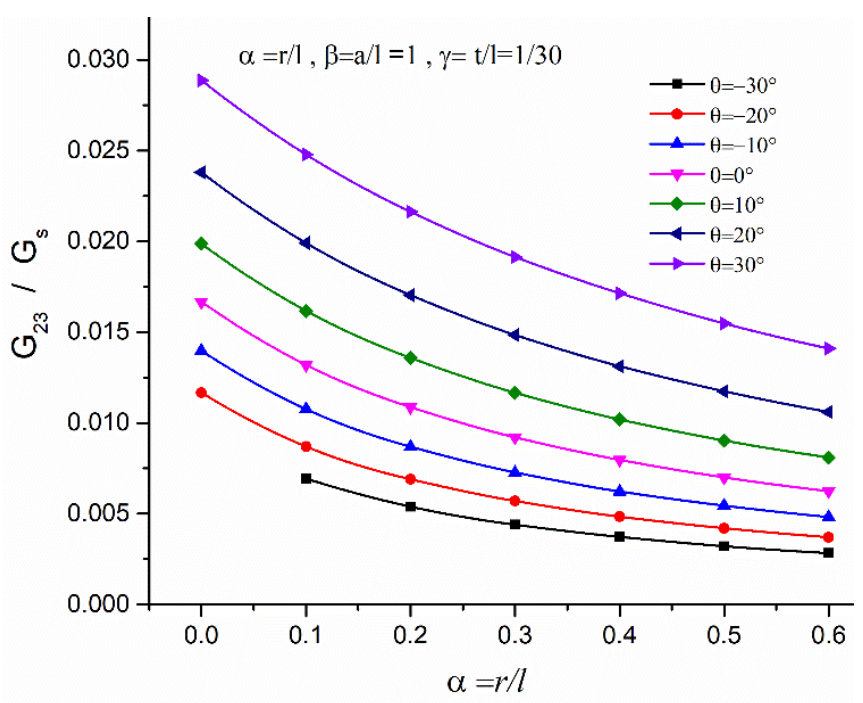

b)

Figure 12. out-of-plane shear modulus versus the curvature ratios $\alpha=r / l$ for different internal cell angle: $\begin{array}{ll}\text { a) } G_{13} / G_{S} & \text { b) } G_{23} / G_{S}\end{array}$

\subsection{Contribution of the transverse bending of the cell walls}

A single value for the transverse shear modulus as versus the cell depth can be calculated using an approach similar to that developed by Grediac [11] and refined by Scarpa and Tomlin [11] and Lira et al. [21] for auxetic centre symmetric configurations. [11,12,21], The same procedure is used here to deduce the out-of-plane mechanical properties of the honeycomb. The transverse shear modulus can be approximated by linking, through the wall cell thickness, the upper ( $G_{23}^{\text {Upper }}$ ) and lower ( $G_{23}^{\text {lower }}$ ) shear limits by equation (29) which is obtained by a non-linear fitting with a $97 \%$ confidence level on 989 configurations. The shear modulus $G_{23}^{r / l}$ can be expressed by:

$G_{23}^{r / l}=G_{23}^{\text {lower }}+f\left(\frac{b}{l}\right)\left(G_{23}^{\text {Upper }}-G_{23}^{\text {lower }}\right)$

With functions for each relative curvature $r / l$. 


$$
\begin{aligned}
& \left.f\left(\frac{b}{l}\right)\right|_{r=0 / 30}=0.01462+1.22273\left(0.57519^{\left(\frac{b}{l}\right)}\right) \\
& \left.f\left(\frac{b}{l}\right)\right|_{r=5 / 30}=0.01497+0.72459\left(0.63557^{\left(\frac{b}{l}\right)}\right) \\
& \left.f\left(\frac{b}{l}\right)\right|_{r=10 / 30}=0.04232+1.09393\left(0.72426^{\left(\frac{b}{l}\right)}\right)
\end{aligned}
$$

By putting (30) in (29) one can obtain:

$$
\begin{aligned}
& G_{23}^{\alpha=0}=G_{23}^{\text {lower }}+\left[0.01462+1.22273\left(0.57519^{\left(\frac{b}{l}\right)}\right)\right]\left(G_{23}^{\text {loper }}-G_{23}^{\text {lower }}\right) \\
& G_{23}^{\alpha=\frac{5}{30}}=G_{23}^{\text {lower }}+\left[0.01497+0.72459\left(0.63557^{\left(\frac{b}{l}\right)}\right)\right]\left(G_{23}^{\text {Upper }}-G_{23}^{\text {lower }}\right) \\
& G_{23}^{\alpha=\frac{10}{30}}=G_{23}^{\text {lower }}+\left[0.04232+1.09393\left(0.72426^{\left(\frac{b}{l}\right)}\right)\right]\left(G_{23}^{\text {Upper }}-G_{23}^{\text {lower }}\right)
\end{aligned}
$$

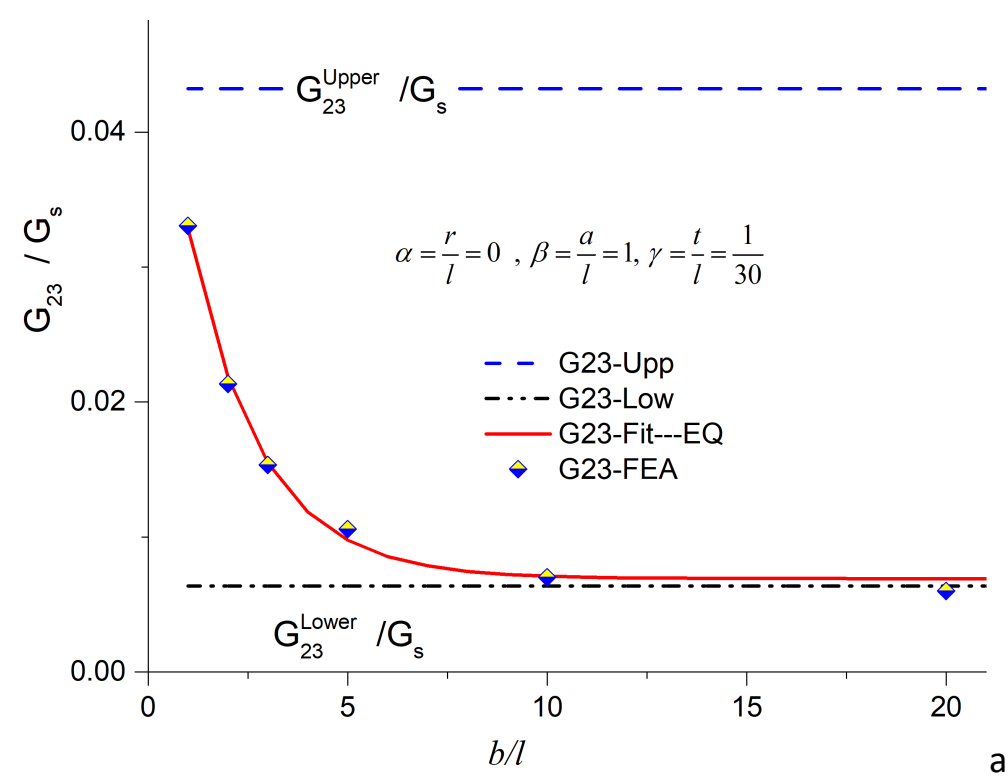




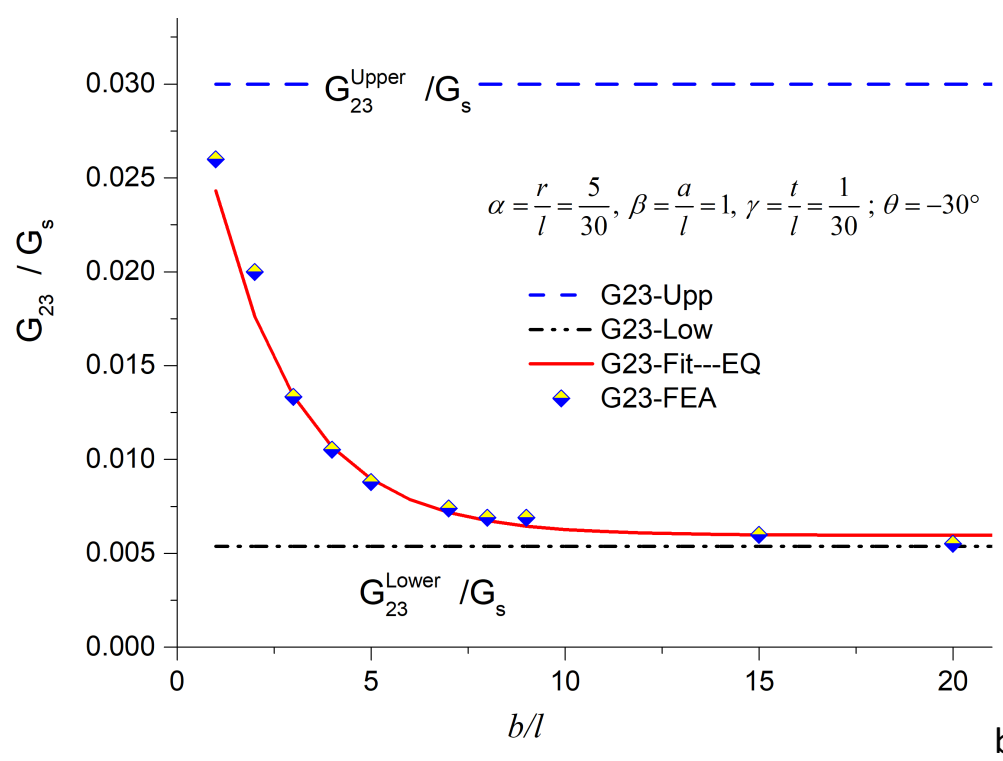

b)

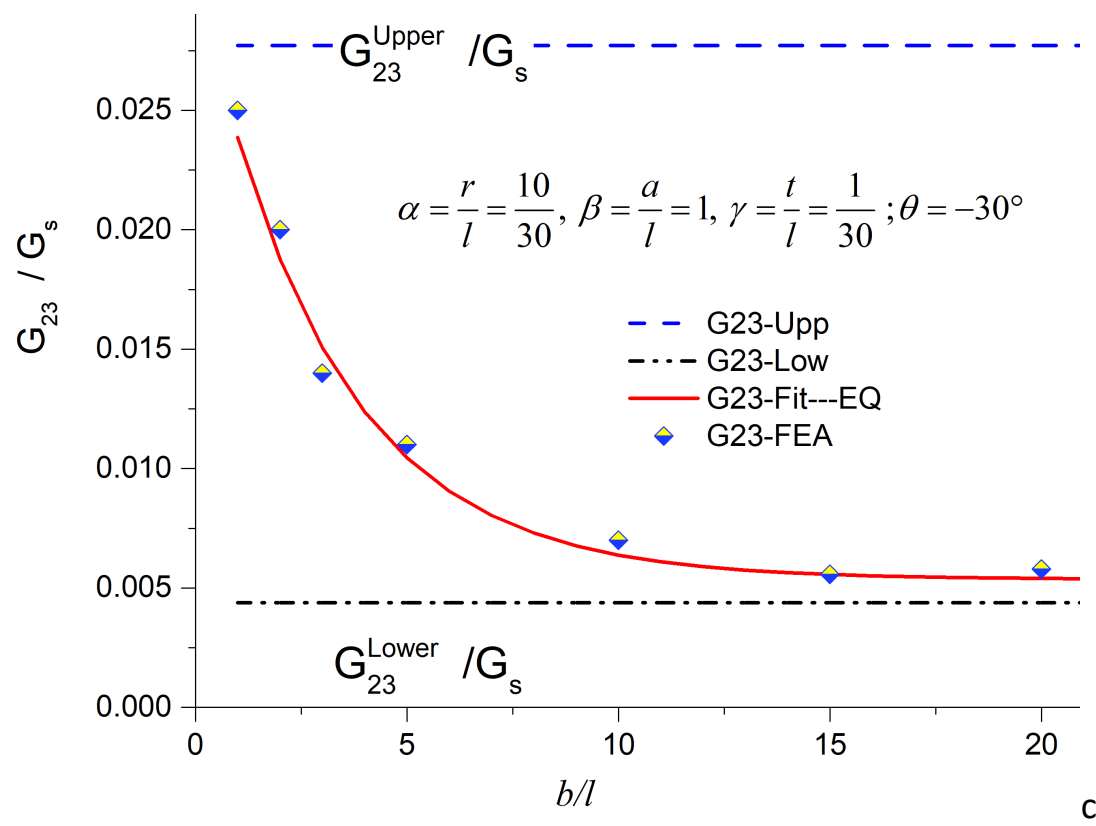

Figure 13. Relative Shear modulus $\mathrm{G}_{23} / \mathrm{G}_{\mathrm{s}}$ versus $b / l$ ratio but with a fixed $\beta=a / l=1$ and $\gamma=t / l=1 / 30$ and $\theta=-30^{\circ}$ and for: a) $\alpha=r / l=0 \quad$ b) $\alpha=r / l=5 / 30 \quad$ c) $\alpha=r / l=10 / 30$.

Figures 13 shows the variation of the $G_{23} / G_{s}$ module versus the effective call depth $b / l$ and for the three curvature radios: $r / l=0,5 / 30$ et $10 / 30$. A significant agreement between the FEM results and the approximate relationship can be observed.

\subsection{Variation of out-of-plane elasticity modulus $E_{3} / E_{s}$ versus the effective geometric} parameters

Figure 14 shows the variation of the non-dimensional transverse Young's modulus $E_{3} / E$ s versus the relative curvature $\alpha=r / l$ and the internal cell angle $\theta$ with maintaining the two ratios $\beta=1$ 
and $\gamma=0.05$ constant, which can also represent the relative density $\rho / \rho_{\mathrm{s}}$. The $E_{3} / E s$ modulus reaches its maximum when the values of $\alpha$ are low for negative cell angles (between -10 to $30)$, it decreases parabolically with the increase of $\alpha$. Moreover, the $E_{3} / E s$ module of the auxetic honeycombs is more sensitive to the $\alpha$ ratio.

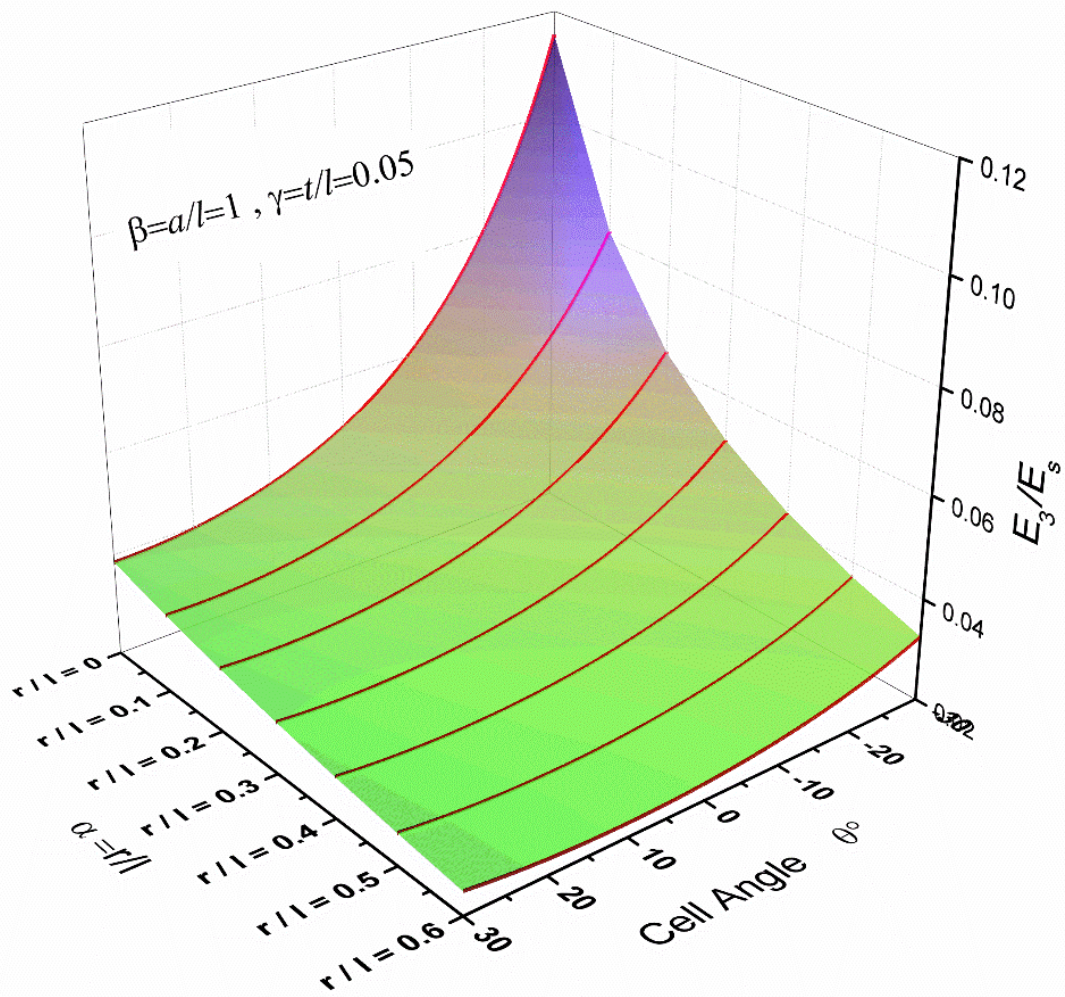

Figure 14. Nondimensional Young's Modulus $E_{3} / E_{s}$ versus curvature ratios $\alpha=r / l$ and internal cell angle $\theta$ for $\beta=1, \gamma=1 / 30$.

Figure 15 represents a comparison between the analytical results (in line) and those obtained by FEA (points). All results are computed by considering a variation versus $\theta$ (Figure 15a) and $r / l$ (Figure 15b) and fixing $\beta=a / l=1$ and $\gamma=t / l=0.05$. The effective modulus $E_{3} / E_{s}$ of the cells represents the relative density and theoretically does not depend on the cell depth. For negative cell angles $\left(-30<\theta<0{ }^{\circ}\right)$ the compressive modulus decreases considerably from 36 to $51 \%$ depending on $r / l$, while for $0<\theta<+30^{\circ}$ this reduction is only from 5 to $31 \%$ (Figure $15-\mathrm{a}$ ). The evolution of $E_{3} / E_{s}$ versus $r / l$ is in parabolic form (Figure 15-b) similar to that of figure 15a. For the extreme curves, a drop of $E_{3} / E_{s}$ by $46 \%$ is recorded when $6.6 \%<r / l<33 \%$ for $\theta$ equal to $30^{\circ}$, this reduction is only around $14 \%$ for $\theta$ equal to $+30^{\circ}$ (Figure 15-b). 


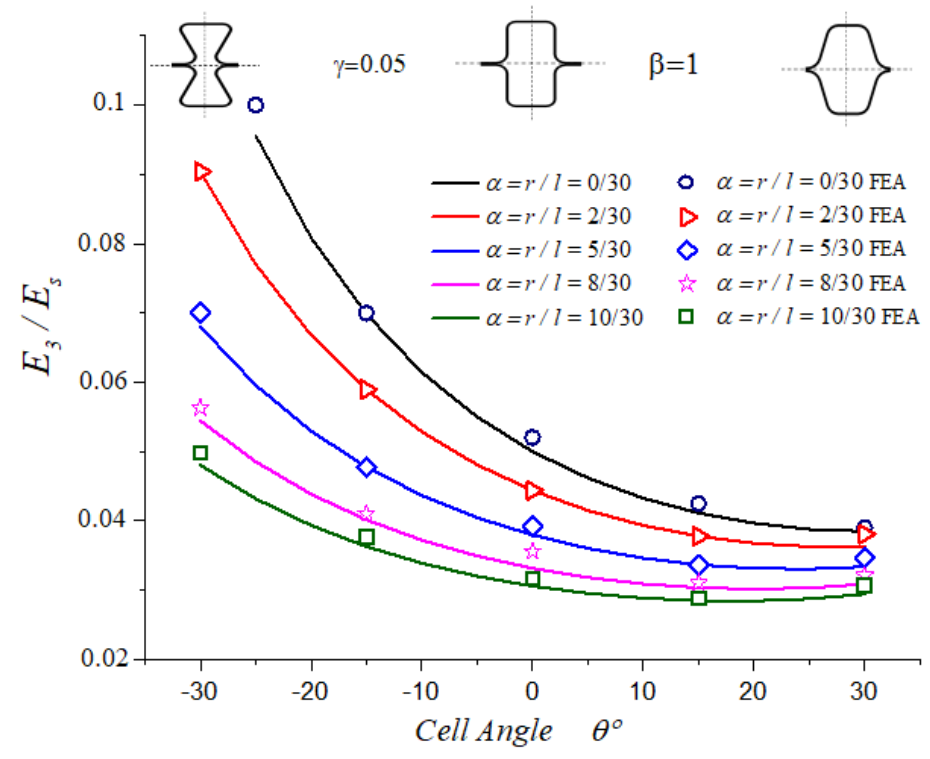

a)

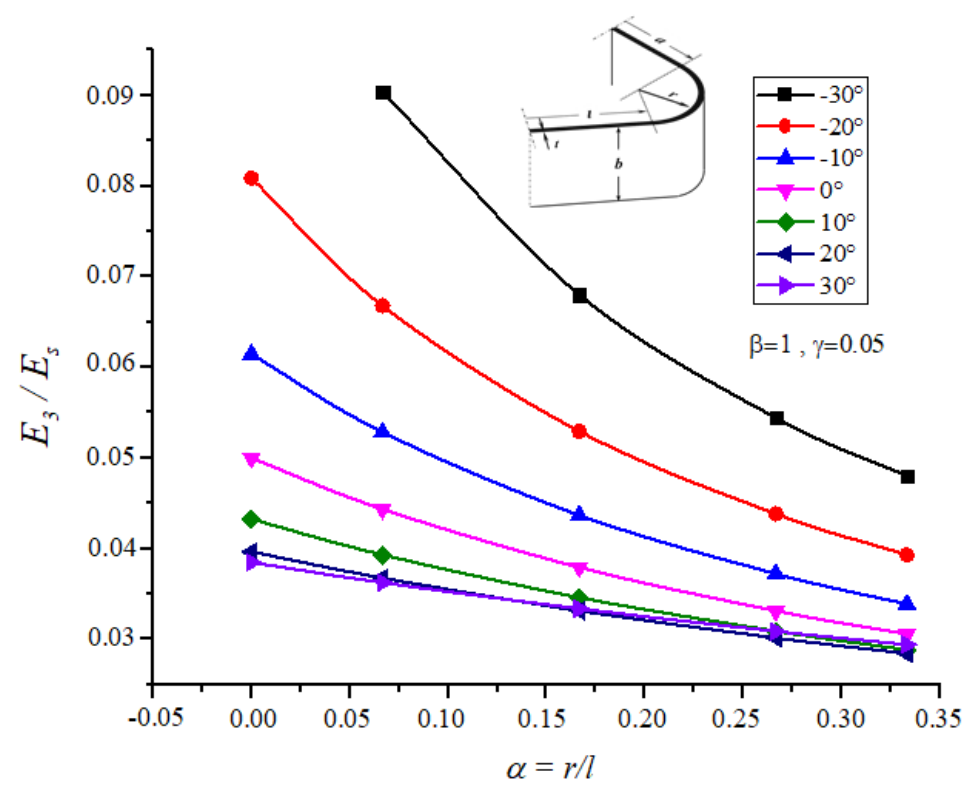

b)

Figure 15. Evolution of $E_{3} / E_{S}$ for a fixed $\beta=1, \gamma=0.05$ versus:

$\begin{array}{lll}\text { a) Internal cell angle } \theta & \text { b) Cell curvature ratios } \alpha=r / l\end{array}$

The out-of-plane nondimensional moduli $G_{13} / G s, G_{23} / G s$ and $E_{3} / E_{s}$ versus $\theta$ show a quasiparabolic form and their respective reductions are $\sim 22 \%, 48.5$ and $\sim 38 \%$ for $-30<\theta<0$ (Figure 17). For $0<\theta<+30$ these reductions become much smaller $\left(7.9 \%\right.$ for $\left.G_{13} / G_{s}\right)$. The nondimensional engineering constants $G_{23} / G_{s}$ and $E_{3} / E_{s}$ have a concave behavior and reach their minimum at $+12^{\circ}$ and $+19^{\circ}$ with reductions of $7.3 \%$ and $8.0 \%$ respectively, then they increase by $12.5 \%$ and $2.9 \%$ for $+30^{\circ}$. For $\theta<0$ the geometry of the cell becomes reentrant 
and the effective Poisson's ratio $v_{12}$ becomes negative. This involves a strong increase in the out-of-plane moduli $G_{13} / G s, G_{23} / G s$ and $E_{3} / E_{s}$ (Figure 16).

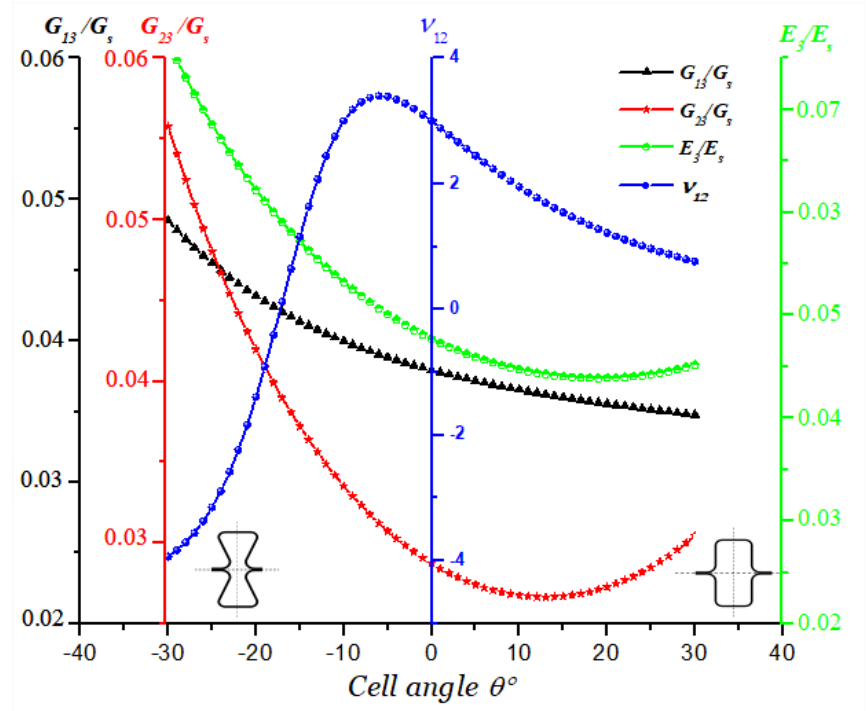

Figure 16. Multi-axis representation of out-of-plane modulus $r / l=0.3$.

\subsection{Variation of $\left(G_{i j} / G_{s}\right) /\left(\rho / \rho_{s}\right)$ versus the cell geometric parameters}

Figure 18 shows the variation of the specific nondimensional shear bounds versus the unit cell parameters. The curves are parametrized for $0<r / l<0.6$ and $-30^{\circ}<\theta<30^{\circ}$.

When the radius of the cell wall is zero the bound $G_{13}\left(G_{13}\right.$ upper/Lower $\left./ G_{s}\right) /\left(\rho / \rho_{\mathrm{s}}\right)$ shows - as expected - a parabolic variation versus the internal cell angle, with a maximum at $\theta=0^{\circ}$ (Figure $17 \mathrm{a})$. Quite interestingly, the $\left(G_{13}\right.$ upper $\left./ G_{s}\right) /\left(\rho / \rho_{\mathrm{s}}\right)$ ratio decreases with increasing $\mathrm{r} / 1$ ratios and shifts the peak value towards positive internal cell angles. One can notice the presence of an internal angle of $\theta=16.88^{\circ}$, for which the nondimensional specific upper bound is the same no matter which radius of curvature is adopted in the cell. Non-zero radiuses of curvature also tend to generate honeycombs with higher nondimensional specific upper bounds within positive internal cell angles. This type of behavior is not specifically observed for the nondimensional specific lower bounds $\left(G_{13}^{\text {Lower }} / G_{s}\right) /\left(\rho / \rho_{\mathrm{s}}\right)$ (Figure $\left.17 \mathrm{~b}\right)$, with only $\sim 2 \%$ maximum differences between positive and negative internal cell angles configurations that depend on the various nondimensional curvatures of the cell walls.

The specific $\left(G_{23}{ }^{U p p e r} / G_{s}\right)$ bound shows a different bevavior, which is expected for these special orthotropic architected materials. When the radius of curvature is zero the distribution of the specific The nondimensional specific upper bound modulus is symmetric with a minimum on to the $\theta=0^{\circ}$ internal cell angle (Figure 18c). Non-zero radiuses of curvature shift the minimum 
towards positive internal cell angles, and auxetic configurations tend to possess higher specific nondimensional trasverse shear stiffnesses. Quite interestingly, the specific nondimensional bound associated to $\left(G_{23}{ }^{\text {Lower }} / G_{s}\right)$ increases monotonically with the internal cell angle, while the sensitivity versus the cell wall radiuses is more pronounced for positive values of $\theta^{\circ}$ (Figure $17 d)$.

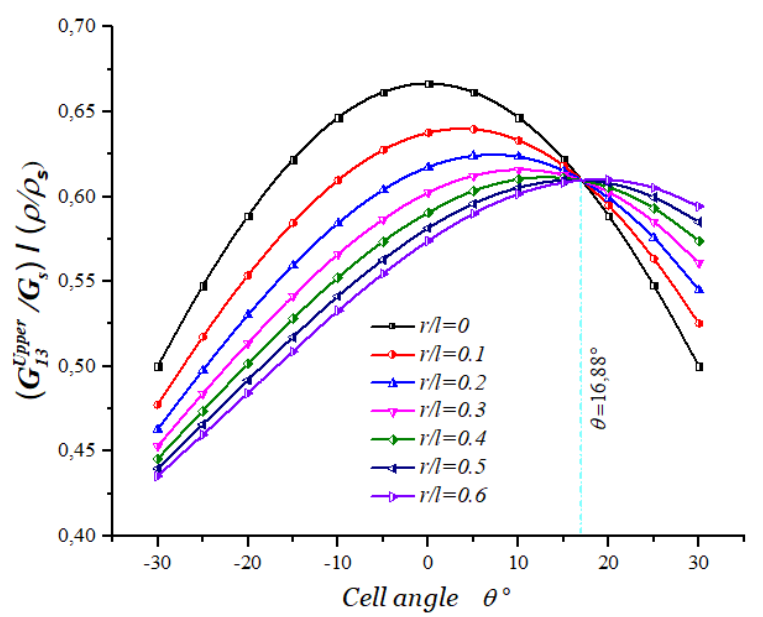

a)

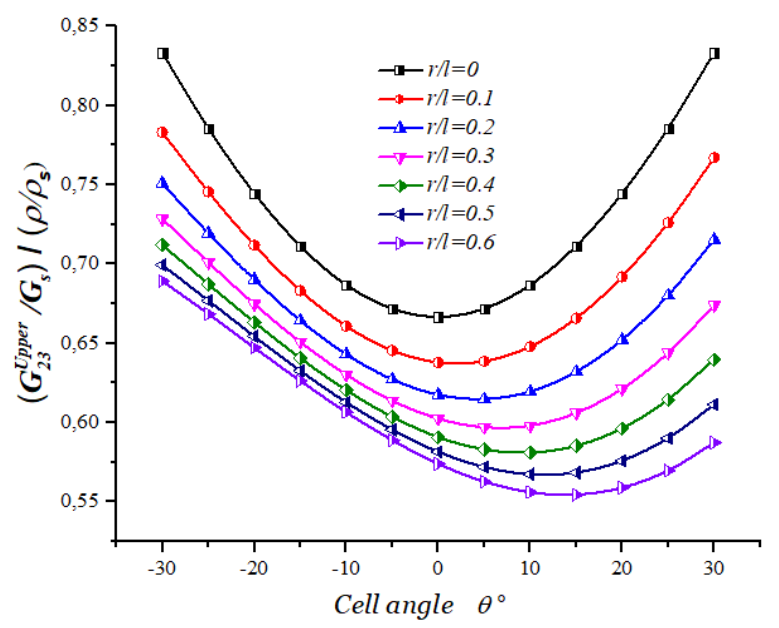

c)

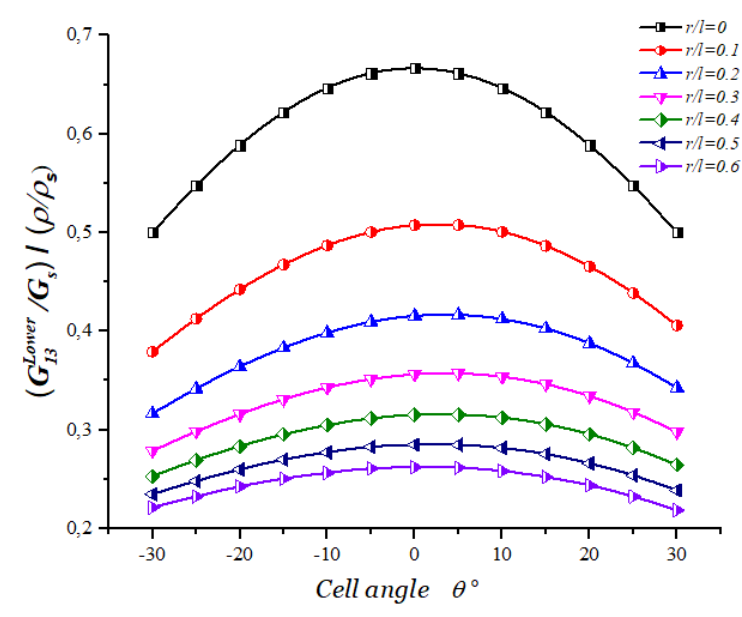

b)

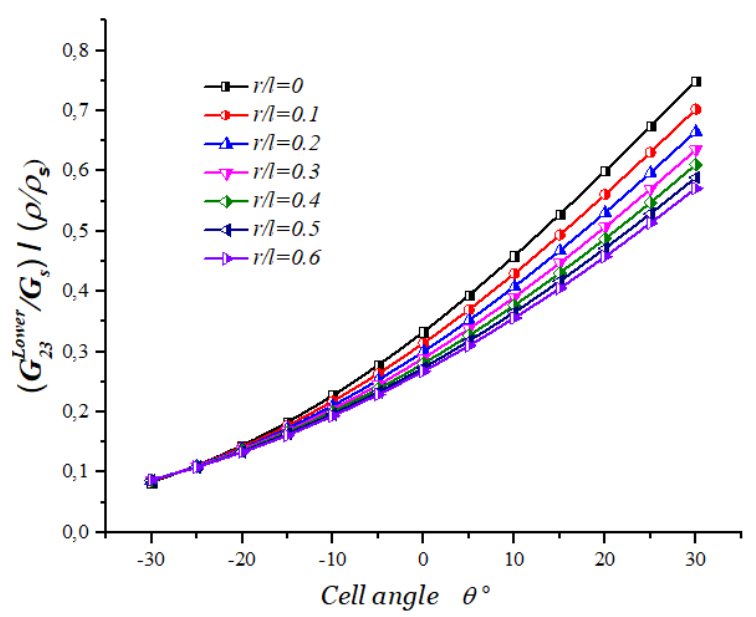

d)

Figure 17. Variation of the ratio $\left(G_{i j} / G_{s}\right) /\left(\rho / \rho_{\mathrm{s}}\right)$ versus the effective geometric parameters for
a) $\left(G_{13}{ }^{\text {upper }} / G_{s}\right) /\left(\rho / \rho_{\mathrm{s}}\right)$
b) $\left(G_{13}{ }^{\text {Lower }} / G_{s}\right) /\left(\rho / \rho_{\mathrm{s}}\right)$
c) $\left(G_{23}{ }^{\text {upper }} / G_{s}\right) /\left(\rho / \rho_{\mathrm{s}}\right)$
d) $\left(G_{23}{ }^{\text {Lower }} / G_{s}\right) /\left(\rho / \rho_{\mathrm{s}}\right)$ 


\section{Conclusion}

This work has shown the behavior of the out-of-plane engineering elastic constants of honeycomb configurations with curved cell walls. The work has described a series of analytical and numerical models parametrized versus the internal geometric features of the lattice materials. The numerical results of this investigation confirm the validity of all the models proposed. These models can be used for design purposes and also provide corrective factors to assess the performance against conventional honeycombs. The results show that the shear modulus $G_{13}$ is maximized for negative cell angles $\theta$, this is particularly advantageous for structures where low weight and thickness accompanied by high stiffness. For a given curvature ratio $\mathrm{r} / \mathrm{l}$, the modulus $G_{23} / G_{s}$ reaches its maximum for the lowest values of the cell depth and for auxetic configurations, while it decreases parabolically with the increase of $\theta$.

The use of 3D representative finite element elementary volume highlights the influence of the cell honeycomb depth. The nondimensional $G_{23} / G_{s}$ modulus $b / l$ decreases exponentially with the depth ratio and stabilizes in the vicinity of the lower limit from $b / l$ equal to 10 . This is also consistent with what observed in other honeycombs with different topologies.

The nondimensional $E_{3} / E s$ modulus reaches its maximum when the values of $\alpha$ are low for negative cell angles (between -30 to -10). The nondimensional transverse modulus also depends on the relative density only, regardless of the depth of the cell. The $E_{3} / E s$ modulus decreases sharply for cell angles between $-30^{\circ}$ to $0^{\circ}$ and then the reduction becomes less pronounced. A $45 \%$ drop of the nondimensional $E_{3} / E_{s}$ modulus was notice when $r / l$ pass from $2 / 30$ to $10 / 30$ for $\theta=-30$ to 0 with fixed values of $\beta=1$ and $\gamma=0.05$. The modulus drops by $21 \%$ only for $\theta=$ +30 .

Some very interesting conclusions can be drawn by looking at the nondimensional specific transverse shear bounds versus internal cell angles and radiuses of cell wall curvature. Specific lower bounds for $G_{23}$ and $G_{13}$ show similar sensivities to honeycombs with no radius of cell wall curvature versus the internal cell angle. The $r / l$ ratio affects the scaling of the specific lower bound values either across the whole internal cell angles range, or only for positive $\theta$ values. On the contrary, the specific upper bounds appear to be much more sensitive to both internal cell angles and especially the cell walls radiuses, with the latter shifting the maximum and minimum values towards positive $\theta$ angles. It is also possible to identify an internal cell angle that provides a specific nondimensional upper modulus independent of the cell wall radius used. 
Upper shear moduli are typical of very thin honeycombs; compounded with the specific inplane elastic properties of the curved cell honeycombs, these data could be useful to design thin sandwich panels with high specific stiffness yet significantly tailorable mechanical cores.

\section{References}

[1] Abd El-Sayed FK, Jones R, Burgess IW. A theoretical approach to the deformation of honeycomb based composite materials. Composites 1979;10:209-14. https://doi.org/10.1016/0010-4361(79)90021-1.

[2] Gibson LJ, Ashby MF, Schajer GS, Robertson CI. The mechanics of two-dimensional cellular materials. Proceedings of the Royal Society of London A Mathematical and Physical Sciences 1982;382:25-42. https://doi.org/10.1098/rspa.1982.0087.

[3] Kolpakov AG. Determination of the average characteristics of elastic frameworks. Journal of Applied Mathematics and Mechanics 1985;49:739-45. https://doi.org/10.1016/0021-8928(85)90011-5.

[4] Masters IG, Evans KE. Models for the elastic deformation of honeycombs. Composite Structures 1996;35:403-22. https://doi.org/10.1016/S0263-8223(96)00054-2.

[5] Bitzer T, Bitzer T. Honeycomb and sandwich testing. Honeycomb Technology, Springer Netherlands; 1997, p. 149-92. https://doi.org/10.1007/978-94-011-5856-5_8.

[6] Josephine Kelvina Florence S, Renji K, Subramanian K. Modal Density of Honeycomb Sandwich Composite Cylindrical Shells Considering Transverse Shear Deformation n.d. https://doi.org/10.20855/ijav.2018.23.11241.

[7] Penzien J, Didriksson T. Effective shear modulus of honeycomb cellular structure. AIAA Journal 1964;2:531-5. https://doi.org/10.2514/3.2346.

[8] Ueng CES, Underwood EE, Liu TL. Shear modulus of superplastically formed sandwich cores 谉. Computers and Structures 1979;10:393-7. https://doi.org/10.1016/00457949(79)90110-X.

[9] Gibson LJ, Ashby MF. Cellular solids: Structure and properties,. Second edi. Cambridge University Press; 1997. https://doi.org/10.1017/CBO9781139878326.

[10] Kelsey S, Gellatly RA, Clark BW. The Shear Modulus of Foil Honeycomb Cores: A Theoretical and Experimental Investigation on Cores Used in Sandwich Construction. Aircraft Engineering and Aerospace Technology 1958;30:294-302. https://doi.org/10.1108/eb033026.

[11] Grediac M. A finite element study of the transverse shear in honeycomb cores. International Journal of Solids and Structures 1993;30:1777-88. https://doi.org/10.1016/0020-7683(93)90233-W.

[12] Scarpa F, Tomlin PJ. On the transverse shear modulus of negative Poisson's ratio honeycomb structures. Fatigue and Fracture of Engineering Materials and Structures 2000;23:717-20. https://doi.org/10.1046/j.1460-2695.2000.00278.x.

[13] Zhang J, Ashby MF. The out-of-plane properties of honeycombs. International Journal 
of Mechanical Sciences 1992;34:475-89. https://doi.org/10.1016/0020-7403(92)900137.

[14] Shi G, Tong P. Equivalent transverse shear stiffness of honeycomb cores. International Journal of Solids and Structures 1995;32:1383-93. https://doi.org/10.1016/00207683(94)00202-8.

[15] Lin TC, Yang MY, Huang JS. Effects of solid distribution on the out-of-plane elastic properties of hexagonal honeycombs. Composite Structures 2013;100:436-42. https://doi.org/10.1016/j.compstruct.2013.01.007.

[16] Malek S, Gibson L. Effective elastic properties of periodic hexagonal honeycombs. Mechanics of Materials 2015;91:226-40. https://doi.org/10.1016/j.mechmat.2015.07.008.

[17] Stefan S, Marin S, Dan Mihai C, Adriana Georgeta S. On the evaluation of mechanical properties of honeycombs by using finite element analyses. INCAS BULLETIN 2015;7:135-50. https://doi.org/10.13111/2066-8201.2015.7.3.13.

[18] Cote F, Deshpande VS, Fleck NA. The shear response of metallic square honeycombs. Journal of Mechanics of Materials and Structures 2006;1:1281-99. https://doi.org/10.2140/jomms.2006.1.1281.

[19] Bezazi A, Scarpa F, Remillat C. A novel centresymmetric honeycomb composite structure. Composite Structures 2005;71:356-64. https://doi.org/10.1016/j.compstruct.2005.09.035.

[20] Bezazi A, Remillat C, Innocenti P, Scarpa F. In-plane mechanical and thermal conductivity properties of a rectangular-hexagonal honeycomb structure. Composite Structures 2008;84:248-55. https://doi.org/10.1016/j.compstruct.2007.08.005.

[21] Lira C, Innocenti P, Scarpa F. Transverse elastic shear of auxetic multi re-entrant honeycombs. Composite Structures 2009;90:314-22. https://doi.org/10.1016/j.compstruct.2009.03.009.

[22] Lira C, Scarpa F, Tai YH, Yates JR. Transverse shear modulus of SILICOMB cellular structures. Composites Science and Technology 2011;71:1236-41. https://doi.org/10.1016/j.compscitech.2011.04.008.

[23] Gong X, Huang J, Scarpa F, Liu Y, Leng J. Zero Poisson's ratio cellular structure for two-dimensional morphing applications. Composite Structures 2015;134:384-92. https://doi.org/10.1016/j.compstruct.2015.08.048.

[24] Balawi S, Abot JL. A refined model for the effective in-plane elastic moduli of hexagonal honeycombs. Composite Structures 2008;84:147-58. https://doi.org/10.1016/j.compstruct.2007.07.009.

[25] Meraghni F, Desrumaux F, Benzeggagh ML. Mechanical behaviour of cellular core for structural sandwich panels. Composites Part A: Applied Science and Manufacturing 1999;30:767-79. https://doi.org/10.1016/S1359-835X(98)00182-1.

[26] Hohe J, Becker W. A refined analysis of the effective elasticity tensor for general cellular sandwich cores. International Journal of Solids and Structures 2001;38:3689-717. https://doi.org/10.1016/S0020-7683(00)00246-8. 
[27] Hohe J, Becker W. An energetic homogenisation procedure for the elastic properties of general cellular sandwich cores. Composites Part B:Engineering 2001;32:185-97. https://doi.org/10.1016/S1359-8368(00)00055-X.

[28] Harkati EH, Daoudi NE-H, Abaidia CE, Bezazi A, Scarpa F. The Elastic Uniaxial Properties of a Center Symmetric Honeycomb with Curved Cell Walls: Effect of Density and Curvature. Physica Status Solidi (B) 2017;254:1600818. https://doi.org/10.1002/pssb.201600818.

[29] Becker W. Closed-form analysis of the thickness effect of regular honeycomb core material. Composite Structures 2000;48:67-70. https://doi.org/10.1016/S02638223(99)00074-4.

[30] SIMULIA. ABAQUS, version CEA 6.10-1 User's Manual. Dassault Systèmes; 2010.

[31] Chamis CC, Aiello RA, Murthy PLN. Fiber composite sandwich thermostructural behavior: computational simulation. Journal of Composites Technology and Research 1988;10:93-9. https://doi.org/10.1520/ctr10135j.

[32] Torquato S, Gibiansky L V., Silva MJ, Gibson LJ. Effective mechanical and transport properties of cellular solids. International Journal of Mechanical Sciences 1998;40:7182. https://doi.org/10.1016/s0020-7403(97)00031-3.

[33] Riccio, A., Raimondo, A., Saputo, S., Sellitto, A., Battaglia, M., Petrone, G. A numerical study on the impact behaviour of natural fibres made honeycomb cores (2018) Composite Structures, 202, pp. 909-916. DOI: 10.1016/j.compstruct.2018.04.062.

[34] Riccio, A., Raimondo, A., Sellitto, A., Acanfora, V., Zarrelli, M.; Multifunctional Polypropylene Core for Aerospace Sandwich Composite Panels (2016) Procedia Engineering, 167, pp. 64-70. DOI: 10.1016/j.proeng.2016.11.670.

[35] Petrone, G., Rao, S., De Rosa, S., Mace, B.R., Franco, F., Bhattacharyya, D.; Behaviour of fibre-reinforced honeycomb core under low velocity impact loading (2013); Composite Structures, 100, pp. 356-362. DOI: 10.1016/j.compstruct.2013.01.004.

[36] Yamashita, M., Gotoh, M.; Impact behavior of honeycomb structures with various cell specifications - Numerical simulation and experiment, (2005) International Journal of Impact Engineering, 32 (1-4), pp. 618-630. DOI: 10.1016/j.ijimpeng.2004.09.001.

[37] Horrigan, D.P.W., Aitken, R.R., Moltschaniwskyj, G., Modelling of Crushing Due to Impact in Honeycomb Sandwiches, (2000) Journal of Sandwich Structures \& Materials, 2 (2), pp. 131-151. DOI: 10.1177/109963620000200203. 